\title{
A Developmental Switch to GABAergic Inhibition Dependent on Increases in Kv1-Type $\mathrm{K}^{+}$Currents
}

\author{
MacKenzie A. Howard, R. Michael Burger, and Edwin W Rubel \\ Department of Physiology and Biophysics and Virginia Merrill Bloedel Hearing Research Center, University of Washington, Seattle, Washington 98195
}

\begin{abstract}
Mature nucleus magnocellularis (NM) neurons, the avian homolog of bushy cells of the mammalian anteroventral cochlear nucleus, maintain high $\left[\mathrm{Cl}^{-}\right]_{\mathrm{i}}$ and depolarize in response to GABA. Depolarizing GABAergic postsynaptic potentials (GPSPs) activate both the synaptic conductance and large outward currents, which, when coupled together, inhibit spikes via shunting and spike threshold accommodation. We studied the maturation of the synaptic and voltage-dependent components of inhibition in embryonic NM neurons using whole-cell and gramicidin-perforated patch-clamp techniques to measure $\mathrm{Cl}^{-}$reversal potential, GABAergic synaptic responses, and voltage-dependent outward currents. We found that GABA enhanced excitability in immature NM neurons, undergoing a switch to inhibitory between embryonic day 14 (E14) and E18. Low-voltage-activated Kv1-type (dendrotoxin-I sensitive) $\mathrm{K}^{+}$currents increased in amplitude between $\mathrm{E} 14$ and $\mathrm{E} 18$, whereas $\mathrm{Cl}^{-}$reversal potential and synaptic conductances remained relatively stable during this period. GABA was rendered inhibitory because of this increase in low-voltage activated outward currents. GPSPs summed with other inputs to increase spike probability at E14. GPSPs shunted spikes at E18, but blocking Kv1 channels transformed this inhibition to excitation, similar to E14 neurons. Subthreshold depolarizing current steps, designed to activate outward currents similar to depolarizing GPSPs, enhanced excitability at E14 but inhibited spiking in E18 neurons. Blocking Kv1 channels reversed this effect, rendering current steps excitatory. We present the novel finding that the developmental transition of GABAergic processing from increasing neuronal excitability to inhibiting spiking can depend on changes in the expression of voltage-gated channels rather than on a change in $\mathrm{Cl}^{-}$reversal potential.
\end{abstract}

Key words: inhibition; development; auditory; magnocellularis; potassium; GABA

\section{Introduction}

GABA activation of $\mathrm{GABA}_{\mathrm{A}}$ receptor $\left(\mathrm{GABA}_{\mathrm{A}} \mathrm{R}\right) \mathrm{Cl}^{-}$currents is the major mode of inhibitory neurotransmission in the mature CNS (McBain and Fisahn, 2001). Factors that shape the response of a neuron to GABA include intracellular $\mathrm{Cl}^{-}$concentration $\left(\left[\mathrm{Cl}^{-}\right]_{\mathrm{i}}\right)$, properties of the $\mathrm{GABA}_{\mathrm{A}} \mathrm{Rs}$, and voltage-dependent properties of the postsynaptic membrane activated by GABAergic postsynaptic potentials (GPSPs). That changes in any one of these properties can alter neural responses to GABA and synaptic integration is well illustrated by the developmental switch from GABAergic excitation to inhibition that many mammalian CNS neurons undergo (Kriegstein and Owens, 2001). For example, in immature hippocampal neurons, chloride reversal potential $\left(E_{\mathrm{Cl}^{-}}\right)$is depolarized relative to action potential (AP) threshold. Under these conditions, GABA is excitatory (Ben-Ari et al., 1989; Cherubini et al., 1990). During maturation, the dominant $\mathrm{Cl}^{-}$ cotransporter expressed in these neurons changes from $\mathrm{Na}^{+}$$\mathrm{K}^{+}-2 \mathrm{Cl}^{-}$cotransporters (e.g., $\mathrm{NKCC} 1$ ) to $\mathrm{K}^{+}-\mathrm{Cl}^{-}$cotransport-

\footnotetext{
Received June 1, 2006; revised Jan. 18, 2007; accepted Jan. 24, 2007.

This work was supported by National Institutes of Health/National Institute on Deafness and Other Communication Disorders Grants DC08035, DC00395, DC03829, DC05361, DC04661, and DC-00466. We thank A. Brumback, Y. Lu, J. Gittelman, H. Brew, D. Sanes, and M. Binder for their valuable insights throughout the course of this study. The authors are thankful for the assistance of A. Brumback and L. Trussell with gramicidin-perforated patch techniques. Correspondence should be addressed to Dr. Edwin W Rubel, Virginia Merrill Bloedel Hearing Research Center, University of Washington, Box 357923, Seattle, WA 98195. E-mail: rubel@u.washington.edu.

R. M. Burger's present address: Department of Biological Sciences, Lehigh University, Bethlehem, PA 18015. DOI:10.1523/JNEUROSCI.5266-06.2007

Copyright $\odot 2007$ Society for Neuroscience $\quad 0270-6474 / 07 / 272112-12 \$ 15.00 / 0$
}

ers (e.g., KCC2). This results in a shift of $E_{\mathrm{Cl}^{-}}$to hyperpolarized values, rendering GABA inhibitory (Rivera et al., 1999). Ge et al. (2006) showed previously that newly generated dentate gyrus granule cells in adult brains also depolarize in response to GABA before undergoing a similar switch. Further, they demonstrated that the early excitatory period is necessary for these neurons to be normally incorporated into the existing circuitry.

In some systems, GPSPs remain depolarizing into maturity, but have an inhibitory effect on the postsynaptic cell (Eccles et al., 1961; Kennedy et al., 1974; Edwards 1990a,b). Mature neurons in nucleus magnocellularis (NM), the avian homolog of mammalian anteroventral cochlear nucleus bushy cells, exhibit such depolarizing, but inhibitory GPSPs (Hyson et al., 1995; Lu and Trussell, 2001). Inhibition in NM is achieved by spike threshold accommodation and shunting of EPSPs (Monsivais and Rubel, 2001). In addition to the shunting effect of the synaptic conductance itself, the depolarizing GPSP activates low-voltage activated (LVA) $\mathrm{K}^{+}$conductances carried mostly by the Kv1-type channels expressed densely in NM (Reyes et al., 1994; Koyano et al., 1996; Rathouz and Trussell, 1998; Fukui and Ohmori, 2004; Lu et al., 2004). Coupling of synaptic and voltage-activated conductances increases the ability of the neuron to inhibit spiking (Monsivais and Rubel, 2001).

In the current study, we explored the developmental relationship between $\left[\mathrm{Cl}^{-}\right]_{\mathrm{i}}$, synaptic conductance, and voltageactivated properties needed for the inhibitory response in NM neurons. We describe the onset and maturation of the GABA 
response as well as the development of $\mathrm{LVA} \mathrm{K}^{+}$conductances using in vitro patch-clamp techniques. GABAergic input in immature NM neurons was found to enhance excitation, followed by conversion to inhibition by embryonic day 18 (E18). However, in contrast to previously described $E_{\mathrm{Cl}^{-}}$-dependent conversion mechanisms, NM neurons show stable $E_{\mathrm{Cl}^{-}}$throughout development. Instead, the switch to inhibition in NM neurons depends on the upregulation of postsynaptic Kv1-type channels. Indeed, inhibition in E18 neurons was transformed into excitation by application of dendrotoxin-I (DTX-I) a specific antagonist of Kv1 channels. These results illustrate an alternative mechanism by which developmental conversion to GABAergic inhibition is achieved.

\section{Materials and Methods}

Slice preparation. We prepared brainstem slices from White Leghorn chicken (Gallus domesticus) embryos at E10, E12, E14, E18, and E21. The entire brainstem was dissected free and isolated in oxygenated low-Ca ${ }^{2+}$ artificial CSF (ACSF) containing the following (in mM): $130 \mathrm{NaCl}, 3 \mathrm{KCl}$, $1.25 \mathrm{NaH}_{2} \mathrm{PO}_{4}, 26 \mathrm{NaHCO}_{3}, 1 \mathrm{MgCl}_{2}, 2 \mathrm{CaCl}_{2}$, and 10 glucose. The $\mathrm{pH}$ of this solution was 7.4, and the osmolarity ranged from 295 to $310 \mathrm{mOsm} / \mathrm{l}$. Identical ACSF solutions were used for experiments on embryos of all ages. The brainstem was blocked coronally, affixed to the stage of a Vibratome (Technical Products International, St. Louis, MO) slicing chamber, and submerged in ACSF. Bilaterally symmetrical coronal slices, 200 $\mu \mathrm{m}$ thick, were made. Slices were then collected in a holding chamber and allowed to equilibrate for $1 \mathrm{~h}$ in ACSF at $36^{\circ} \mathrm{C}$. All slices chosen for the experiments reported here were from the middle of the rostrocaudal extent of NM. Hence, information on developmental gradients along the tonotopic dimension of NM (Rubel et al., 1976; Jackson et al., 1982) is not available in our analyses.

Slices were transferred from the holding chamber to a $0.5 \mathrm{ml}$ recording chamber mounted on a Zeiss (Oberkochen, Germany) Axioskop FS for electrophysiological experiments. This microscope was equipped with a $40 \times$ water-immersion objective and infrared differential interference contrast optics. The recording chamber was superfused continuously with warm $\left(35^{\circ} \mathrm{C}\right)$, oxygenated ACSF (contents identical to slicing solution but with $3 \mathrm{~mm} \mathrm{CaCl}$ ) at a rate of $1-2 \mathrm{ml} / \mathrm{min}$.

Whole cell electrophysiology. Patch pipettes were pulled to resistances of 3-7 M $\Omega$. Current-clamp experiments were performed with an Axoclamp 2B amplifier (Molecular Devices, Foster City, CA). In current-clamp experiments, recording pipettes were filled with $\mathrm{K}^{+}$-based internal solution containing the following (in $\mathrm{mm}$ ): $105 \mathrm{~K}$-gluconate, $35 \mathrm{KCl}, 1$ $\mathrm{MgCl}_{2}, 10$ HEPES-K ${ }^{+}, 5$ EGTA, 4 ATP- $\mathrm{Mg}^{+2}$, and $0.3 \mathrm{GTP}-\mathrm{Na}^{+}$. The junction potential for this solution was calculated to be $-7 \mathrm{mV}$. The junction potential for this and all other solutions was corrected for before data analysis. Voltage-clamp experiments were performed with an Axoclamp 200B amplifier (Molecular Devices). For experiments in which $\mathrm{GABA}_{\mathrm{A}} \mathrm{R}$ currents were recorded, pipettes were filled with a $\mathrm{Cs}^{+}$-based solution containing the following (in $\mathrm{mM}$ ): $70 \mathrm{CH}_{3} \mathrm{CsO}_{3} \mathrm{~S}, 85$ sucrose, 5 BAPTA [bis (o-aminophenoxy)- $N, N, N^{\prime}, N^{\prime}$-tetraacetic acid], 10 HEPES, $4 \mathrm{NaCl}, 1 \mathrm{MgCl}_{2}$, and $1 \mathrm{ATP}$. The junction potential for this solution was calculated to be $-16 \mathrm{mV} . \mathrm{K}^{+}$-current experiments were performed using the $\mathrm{K}^{+}$-based pipette solution $\left(\mathrm{Cs}^{+}\right.$free) described above. Series resistance was compensated for by $85-90 \%$ in all voltage-clamp recordings.

Pipettes were visually guided to NM. NM neurons were easily identified and distinguished from surrounding tissue by cell morphology and location of the nucleus within the slice. After G $\Omega$ seals were attained, membrane patches were ruptured and neurons were held in whole-cell configuration at $I=0$ in current clamp or $-70 \mathrm{mV}$ in voltage clamp. Neurons with depolarized membrane potential (more than $-58 \mathrm{mV}$ ) were excluded from this study. Extracellular stimulation was accomplished using a concentric bipolar electrode (Frederick Haer, Bowdoinham, ME) with a $200 \mu \mathrm{m}$ tip diameter. The electrode was inserted into the slice on the dorsolateral edge of NM. Stimuli consisted of 100- $\mu$ sduration square electric pulses, delivered via a stimulus isolator (1850 A) and interval generator (1830; WP Instruments, New Haven, CT). Pulse intensity was adjusted up from 5 to $50 \mathrm{~V}$ until a postsynaptic response was elicited. Stimulus intensity was then adjusted such that postsynaptic events were of maximal amplitude with minimal failure rate yet antidromic action potentials were not induced.

The $\mathrm{GABA}_{\mathrm{A}} \mathrm{R}$ agonist muscimol was puff applied with a picospritzer (General Valve, Fairfield, NJ). Puff pipettes with a tip diameter of $2-5 \mu \mathrm{m}$ were pulled using the same techniques as for patch pipettes. These were filled with ACSF containing $500 \mu \mathrm{M}$ muscimol. Puff pipettes were visually lowered into close proximity $(10-50 \mu \mathrm{m})$ of the neuron from which recordings were being made. Pressure pulses $20 \mathrm{~ms}$ in duration were used to puff muscimol onto the neuron. Pressure pulses of 10-20 psi were adequate to induce a response without damaging the neuron or disrupting the patch pipette seal.

Gramicidin-perforated patch-clamp electrophysiology. Gramicidinperforated patch recordings were made from E14 and E18 neurons to measure the reversal potential for GABA. These experiments made use of the same pipettes and instrumentation as whole-cell voltage-clamp recordings. Gramicidin was dissolved in DMSO then added to a pipette solution containing $150 \mathrm{~mm} \mathrm{KCl}$ and $10 \mathrm{~mm}$ HEPES; the final gramicidin concentration was $50-80 \mu \mathrm{g} / \mathrm{ml}$. The junction potential for this solution was calculated to be $-4 \mathrm{mV}$. Pipettes were tip-filled with gramicidin-free pipette solution, then backfilled with gramicidin solution. Once a G $\Omega$ seal was established, neurons were held until series resistance stabilized at $<50 \mathrm{M} \Omega$, usually 25-45 min after contact with the neuron. Neurons were held in voltage-clamp mode at a holding potential of $-64 \mathrm{mV}$. $E_{\mathrm{GABA}}$ was measured by stepping the holding potential from -84 to -14 $\mathrm{mV}$ in $10 \mathrm{mV}$ steps of $100 \mathrm{~ms}$ duration. Neurons were held at each potential for $10 \mathrm{~s}$ before muscimol application such that voltagedependent conductances and, thus, holding current, were stabilized. Muscimol was applied using a picospritzer, as described above. To avoid the potential confound of $\mathrm{Cl}^{-}$loading or unloading by the $\mathrm{GABA}_{\mathrm{A}} \mathrm{R}$ current, voltage steps were separated by $60 \mathrm{~s}$. Peak muscimol-induced current was plotted against step potential and linear regression was used to predict the reversal potential. This protocol was repeated in full at least two times in each neuron to ensure repeatability. Neurons that exhibited instability in measured current reversal potential were eliminated from additional analyses. The high $\mathrm{Cl}^{-}$concentration of the pipette solution allowed us to monitor the integrity of the perforated membrane patch. If the patch was disrupted and a whole-cell configuration was established, the reversal potential for $\mathrm{Cl}^{-}$became positive (intracellular $\left[\mathrm{Cl}^{-}\right], 150$ mM; extracellular $\left[\mathrm{Cl}^{-}\right.$], $141 \mathrm{~mm} ; \mathrm{Cl}^{-}$reversal potential, $+1.6 \mathrm{mV}$ ) such that $\mathrm{GABA}_{\mathrm{A}} \mathrm{R}$ currents evoked at all holding potentials in this protocol were inward.

Data analysis. All recording protocols were written and run using the Axograph acquisition and analysis software (version 4.5; Molecular Devices). Leak subtraction was used offline on $\mathrm{K}^{+}$currents using the response to the hyperpolarizing step from -70 to $-75 \mathrm{mV}$ as a baseline. Junction potentials were corrected for in all cases. Statistical analyses $(t$ tests, ANOVAs, and post hoc tests) were performed using StatView version 4.5. The standard for significant differences was defined as $p<0.05$. All graphic presentations of data illustrate mean \pm SEM; $n$ values are presented in the figure legends.

Drugs and chemicals. All bath-applied drugs were allowed to perfuse through the recording chamber for 2 min before subsequent recordings. The following concentrations of each were used in the external solution: $100 \mu \mathrm{M}$ DL-2-amino-5-phosphonopentanioc acid (AP5), $50 \mu \mathrm{M}$ bicuculline methiodide (Bic), $50 \mu \mathrm{M}$ 6,7-dinitroquinoxaliine-2,3(1H,4H)dione (DNQX), $0.1 \mu \mathrm{M}$ DTX-I, $1 \mu \mathrm{M}$ tetrodotoxin (TTX), and $50 \mu \mathrm{M} \mu \mathrm{M}$ 4(N-ethyl- $N$-phenylamino) 1,2-dimethyl-6-(methylamino) pyridinium chloride (ZD-7288). TTX and DTX-I were obtained from Alomone Labs (Jerusalem, Israel). All other drugs and chemicals were obtained from Sigma (St. Louis, MO).

\section{Results}

\section{Onset of $\mathrm{GABA}_{\mathrm{A}} \mathrm{R}$ responses}

$\mathrm{GABA}_{\mathrm{A}}$ Rs carry the depolarizing $\mathrm{Cl}^{-}$currents that inhibit spiking in mature NM neurons. We demonstrated the presence of physiologically functional $\mathrm{GABA}_{\mathrm{A}}$ Rs by puff application of the 


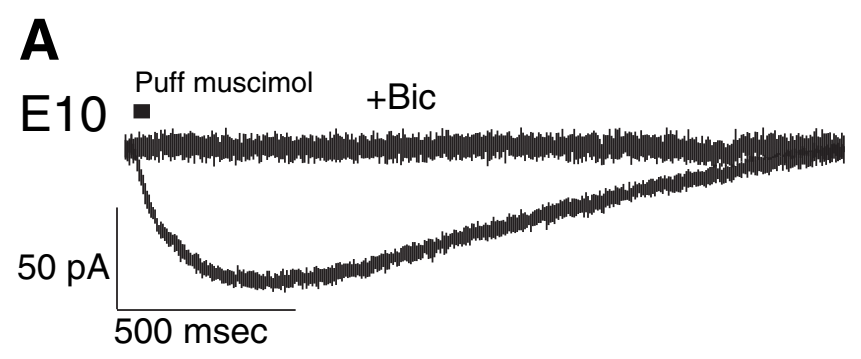

B Puff muscimol

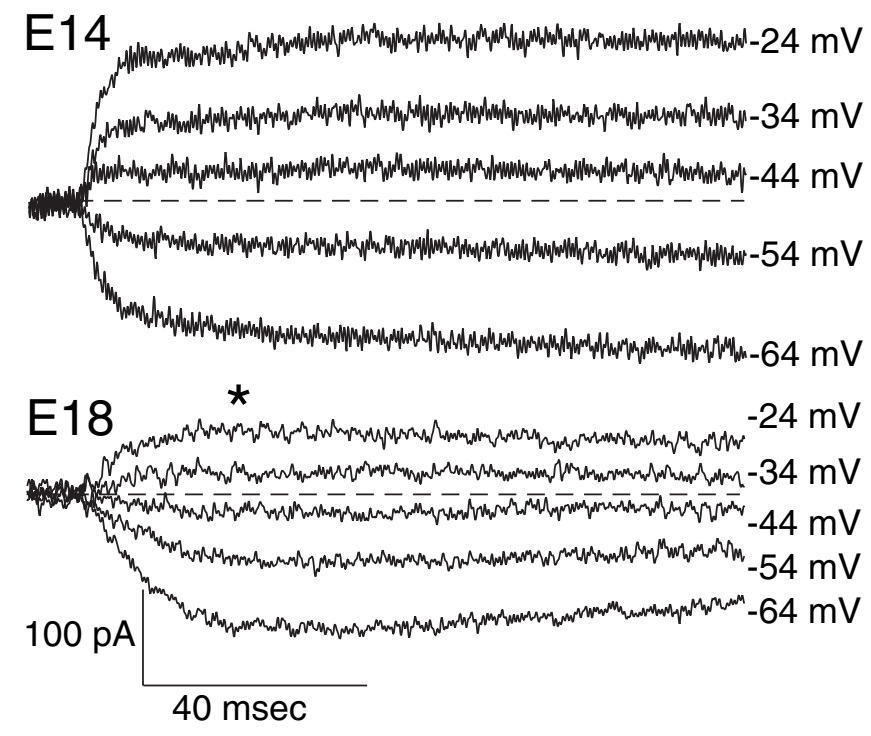

Figure 1. $G A B A_{A} R$ currents can be induced in NM neurons as early as E10. In all experiments, $G A B A_{A} R$ currents were induced with picospritz application of the $G A B A_{A} R$ agonist muscimol in the presence of DNQX and AP5. $A$, Averaged responses to muscimol from a representative E10 neuron held in whole-cell voltage clamp at $-60 \mathrm{mV}$. Dark bars above the traces illustrate the time course of the muscimol puff. These currents were blocked completely with bath application of Bic. $\boldsymbol{B}, G A B A_{A} R$ currents recorded using gramicidin-perforated patch methods from an E14 (top) and E18 neuron. Puffs of muscimol were delivered after holding the neuron for $10 \mathrm{~s}$ at voltage steps ranging from -84 to $-14 \mathrm{mV}$ in $10 \mathrm{mV}$ steps. For clarity, only responses to steps ranging from -64 to $-24 \mathrm{mV}$ are illustrated (numbers to the right label the holding potential for each trace). The asterisk indicates the approximate peak of the E18 traces, at which point measurements of reversal potential were made. Dashed lines indicate the baseline. Trace baseline is adjusted to the average current during the $5 \mathrm{~ms}$ period just before muscimol application. The calibration at the bottom applies to both the E14 and E18 traces. Evoked currents were slow to decay but return to baseline after several seconds (data not shown). GABA $R$ current reversal potential was $-41.8 \pm 1.4 \mathrm{mV}(\mathrm{SEM})$ for E14 $(n=6)$ and $-37.9 \pm 2.2 \mathrm{mV}$ for E18 $(n=4)$.

$\mathrm{GABA}_{\mathrm{A}} \mathrm{R}$ agonist muscimol to voltage-clamped neurons. Robust $\mathrm{GABA}_{\mathrm{A}} \mathrm{R} \mathrm{Cl}^{-}$currents were recorded in all neurons tested, ages E10 and older. Figure $1 A$ illustrates averaged muscimol-induced currents recorded from an E10 neuron. These currents were blocked by bath application of Bic, further confirming that $\mathrm{GABA}_{\mathrm{A}} \mathrm{Rs}$ were responsible for these currents (Chebib and Johnston, 1999). Thus, functional $\mathrm{GABA}_{\mathrm{A}}$ Rs are present as early as E10, before which NM neurons are difficult to reliably identify in our brainstem slices.

Mature NM neurons maintain a higher $\left[\mathrm{Cl}^{-}\right]_{\mathrm{i}}$ than typical CNS neurons and, thus, depolarize in response to GABA (Monsivais and Rubel, 2001). We used gramicidin-perforated patch techniques, which do not disrupt native $\left[\mathrm{Cl}^{-}\right]_{\mathrm{i}}$ (Akaiki, 1996), to determine $E_{\mathrm{GABA}}$ across a range of embryonic development in NM neurons. The holding potential of voltage-clamped neurons $(-64 \mathrm{mV})$ was stepped to a test potential for $12 \mathrm{~s}$. Test potentials ranged from -84 to $-14 \mathrm{mV}$ in $10 \mathrm{mV}$ steps. Ten seconds into this voltage step, muscimol was puff applied as described above. Figure $1 B$ illustrates the $\mathrm{GABA}_{\mathrm{A}} \mathrm{R}$ currents evoked by this protocol in an E18 neuron. The mean \pm SD reversal potential for muscimol currents was $-41.8 \pm 5.7 \mathrm{mV}$ in E14 neurons $(n=17)$ and $-37.9 \pm 6.7 \mathrm{mV}$ in $\mathrm{E} 18(n=9)$ neurons. These values are not significantly different $(p=0.07 ; t$ test $)$, indicating that $E_{\mathrm{GABA}}$ is relatively stable across this period of embryonic development. Thus, GPSPs resulting from activation of $\mathrm{GABA}_{\mathrm{A}}$ Rs are depolarizing at both of these ages.

Monsivais and Rubel (2001) reported a value of $-36 \pm 5.1$ $\mathrm{mV}$ for $E_{\mathrm{GABA}}$ using $\mathrm{E} 21$ and posthatch day 1 (P1) neurons. $\mathrm{Lu}$ and Trussell (2001) reported $E_{\mathrm{GABA}}$ as $-25 \pm 5 \mathrm{mV}$ in neurons ranging from E17 to P10. Although the ionic components of extracellular solutions used in the present report were slightly different from those used in previous studies, this cannot completely account for the discrepancy between our results and those published by Lu and Trussell (2001). The ACSF used during our recordings contains an $\left[\mathrm{Cl}^{-}\right]_{\mathrm{o}}$ of $141 \mathrm{~mm}$, compared with 153 mM used by Lu and Trussell (2001). Taken alone, this difference would make our $E_{\mathrm{GABA}}$ measurements $\sim 2.2 \mathrm{mV}$ more positive than those made by Lu and Trussell (2001). Another difference between our experimental conditions and those of other labs was the use of $\mathrm{HCO}_{3}^{-}$or HEPES as the extracellular buffer. Our experiments used $\left[\mathrm{HCO}_{3}^{-}\right]_{\mathrm{o}}=26 \mathrm{~mm}$, whereas Lu and Trussell (2001) used $\mathrm{HCO}_{3}^{-}$-free HEPES-buffered ACSF. Using the $\mathrm{GABA}_{\mathrm{A}} \mathrm{R} \mathrm{HCO}_{3}^{-} / \mathrm{Cl}^{-}$permeability ratio of 0.18 (Bormann et at., 1987), assuming a physiological $\left[\mathrm{HCO}_{3}^{-}\right]_{\mathrm{i}}=16 \mathrm{mM}$ (Staley et al., $1995)$, we calculated the $E_{\mathrm{HCO}}-$ for our conditions to be $-10 \mathrm{mV}$. Using a $\mathrm{HCO}_{3}^{-}$-free HEPES-based ACSF (Lu and Trussell, 2001) resulted in an $E_{\mathrm{HCO}^{-}}$that is positive and very large. However, because of the low permeability ratio of $\mathrm{HCO}_{3}^{-} / \mathrm{Cl}^{-}$through $\mathrm{GABA}_{\mathrm{A}} \mathrm{Rs}$, these currents will contribute $<3 \mathrm{mV}$ to the difference between our results and those of Lu and Trussell (2001). Again, this does not fully account for the discrepancy between our results and those published previously. We can briefly speculate on other mechanisms that may account for some or all of the difference between our measurements of $E_{\mathrm{GABA}}$ and those of $\mathrm{Lu}$ and Trussell (2001). The lack of extracellular $\mathrm{HCO}_{3}^{-}$ions may alter normal ion transport, such as by the $\mathrm{Na}^{+}-\mathrm{Cl}^{-}-\mathrm{HCO}_{3}^{-}$exchangers of the solute carrier-4 family, disrupting intracellular $\mathrm{Cl}^{-}$and $\mathrm{HCO}_{3}^{-}$concentrations. Chickens are not an inbred strain and come from different suppliers, so genetics may play some role in this difference as well. Neither Monsivais and Rubel (2001) nor $\mathrm{Lu}$ and Trussell (2001) report the values for the junction potentials present during gramicidin experiments, but both of these papers corrected for these values. Differences in the measurement and calculation of junction potentials could also contribute to the differences in reported $E_{\mathrm{GABA}}$. Each of these possibilities should be viewed as a hypothesis and we cannot provide data that supports or refutes any of them. However, each of these papers clearly shows that GPSPs are depolarizing in NM neurons, and that the polarity of GPSPs is relatively stable over time. Thus, the discrepancy between our data and that of Lu and Trussell (2001) does not affect the overall interpretation of our results.

Whereas $E_{\mathrm{GABA}}$ is depolarized compared with resting membrane potential at both these ages, it is hyperpolarized compared with AP voltage threshold at E14 (Table 1). AP voltage threshold was defined as the voltage at which the upward inflection point of the voltage trace occurs. The second derivative of the voltage trace was examined for a negative to positive $x$-axis crossing that indicated an upward inflection point in the original voltage trace. 
Table 1. Resting membrane potential, AP voltage threshold, and $E_{\mathrm{GABA}}$ in millivolts \pm SD $(n)$ in immature and mature NM neurons under various conditions

\begin{tabular}{lll}
\hline & E14 & E18 \\
\hline Resting membrane potential & $-65.4 \pm 2.0(8)$ & $-67.3 \pm 1.3(8)$ \\
AP voltage threshold & $-33.5 \pm 2.9(8)$ & $-37.0 \pm 4.0(8)$ \\
+DTX-I & $-40.8 \pm 4.5(8)$ & $-41.2 \pm 1.6(8)$ \\
\hline
\end{tabular}

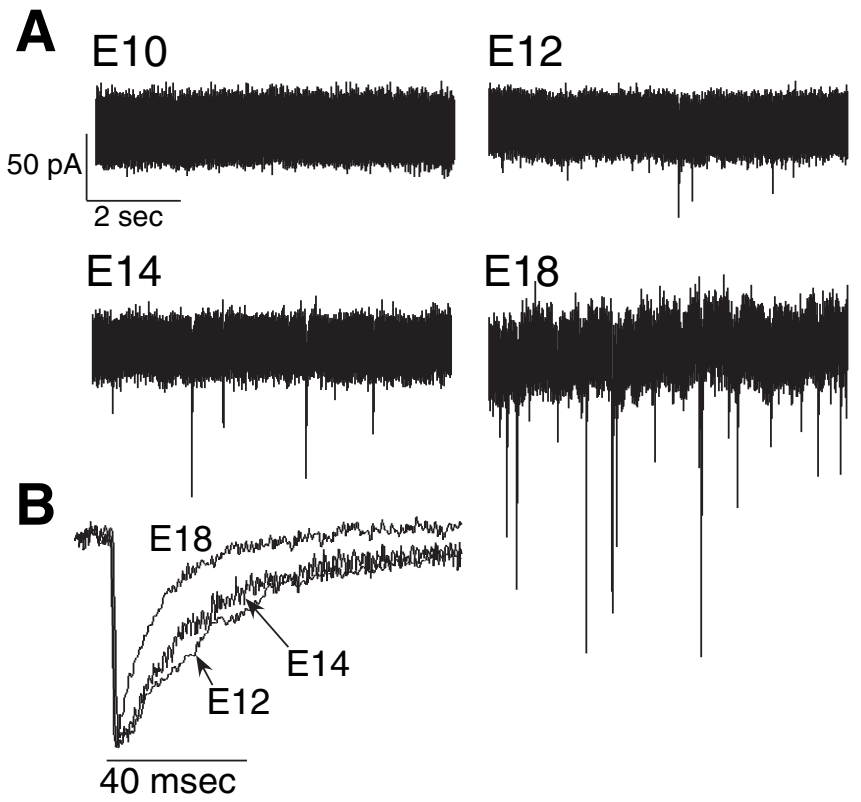

Figure 2. Properties of mGPSCs change during development in NM neurons. All mGPSPs were recorded at a holding potential of $-70 \mathrm{mV}$ during bath application of DNQX and AP5. A, Traces recorded from E10, E12, E14, and E18 show that mGPSC activity first recorded at E12. The E12 trace should not be considered representative (see Results). B, Expanded view of overlaid, averaged $\mathrm{mGPSC}$, normalized in amplitude. These traces illustrate the difference in rise and decay times for $\mathrm{mGPSC}$ at these ages.

Thus, GABA cannot be defined as an excitatory neurotransmitter at this age. At E18, $E_{\mathrm{GABA}}$ and $\mathrm{AP}$ voltage threshold are statistically indistinguishable. $\mathrm{Lu}$ and Trussell (2001) reported GABAevoked APs in NM neurons and, thus, describe GABA as a mixed excitatory/inhibitory transmitter in this system.

\section{Onset of spontaneous GABAergic activity}

Spontaneous release of GABAergic vesicles from presynaptic terminals is detected postsynaptically in the form of miniature GABAergic postsynaptic currents (mGPSCs). We measured mGPSCs to determine the onset and characteristics of GABAergic synaptic currents across development in NM neurons. Figure $2 \mathrm{~A}$ illustrates voltage-clamp chart recordings of mGPSCs from neurons at ages E10, E12, E14, and E18. These traces are representative for all ages except E12. At this age, mGPSCs (mGPSCs) were very infrequent $(<1 / \mathrm{min})$ and were present in only $2 / 11$ neurons. Hence, a trace illustrating mGPSCs was intentionally selected. In all of these experiments, the glutamate receptor antagonists DNQX and AP5 were bath applied throughout these recordings, which were made over several minutes to ensure that an adequate sample was taken from each neuron. No mGPSCs were recorded when Bic was bath applied, but mGPSC activity resumed within minutes of Bic washout (data not shown). No mGPSCs were evident in successful recordings from 13 neurons at ages E10 and E11. The earliest age at which we observed any mGPSCs was E12, where a small proportion (2/11) of neurons exhibited such events. In these neurons, mGPSC frequency was much lower than at older ages $(>0.01 \mathrm{~Hz}$ at E12 vs $\sim 0.8 \mathrm{~Hz}$ at E18), with only a few events detected during recordings several minutes in length. In a subset of neurons at E10, E12, and E14, ruthenium red $(100 \mu \mathrm{M})$ was added to the ACSF to increase the probability of vesicular release and, thus, increase mGPSC frequency (Chang et al., 2003). This manipulation increased mGPSC frequency in E14 and E18 neurons, but failed to elicit recordable mGPSCs in E10 and E11 neurons or in unresponsive E12 neurons (data not shown). However, the possibility remains that spontaneous GABAergic synaptic events may be occurring in these neurons, with frequency and amplitudes too low to detect in our recordings. Figure $2 B$ shows averaged mGPSCs from individual neurons at E12, E14, and E18, normalized in amplitude and overlaid to illustrate typical differences in kinetics.

We made statistical comparisons of mGPSC frequency, current amplitude, $20-80 \%$ rise time, and decay time constant between E14 and E18 age groups. Group data for E12 neurons was not included in these analyses because the extremely low frequency of these events made statistical comparisons inappropriate. The trend of increasing mGPSC frequency between E14 and E18 fell short of a significant difference because of high variability $(p<0.1)$. mGPSC amplitude increased significantly between E14 and E18 $(\mathrm{E} 14,68.8 \pm 31 \mathrm{pA}, n=11 ; \mathrm{E} 18,111 \pm 30 \mathrm{pA}, n=$ $13 ; p<0.01)$. Rise time became faster as neurons matured, decreasing significantly from E14 to E18 (E14, $0.482 \pm 0.16 \mathrm{~ms}, n=$ $11 ; \mathrm{E} 18,0.327 \pm 0.085 \mathrm{~ms}, n=13 ; p<0.01)$. Decay time constant also became faster in more mature neurons, decreasing significantly between these two ages (E14, $19.05 \pm 4.19 \mathrm{~ms}, n=11$; E18, $12.73 \pm 4.83 \mathrm{~ms}, n=13 ; p<0.005)$. Overall, the changes in amplitude and kinetics resulted in larger, faster GABAergic mGPSCs as neurons matured.

\section{Onset of evoked GABAergic responses}

We also studied evoked GPSCs in NM neurons across the same developmental period as mGPSCs. Figure $3 A$ illustrates evoked GPSCs recorded from NM neurons at E10, E12, E14, and E18. The earliest age at which GABAergic postsynaptic responses were evoked was E14; most (93\%) E14 neurons exhibited evoked GPSCs, and all E18 neurons exhibited GPSCs. Panels for E14 and E18 show the block of GPSCs by Bic and recovery after washout. Figure $3 B$ shows averaged GPSCs from E14 and E18 normalized in amplitude and overlaid such that the kinetics of the currents can be compared. Again, statistical comparisons of current amplitude, $20-80 \%$ rise time, and decay time constant were made between E14 and E18 age groups. These analyses revealed a significant decrease in decay time constant from E14 to E18 (E14, $23.47 \pm 5.05 \mathrm{~ms}, n=9$; E18, $15.82 \pm 5.07, n=13 ; p<0.005)$. No significant changes were seen in amplitude or rise time of GPSCs across age groups. The change in decay time constant resulted in faster, briefer GPSCs. The net result is similar to the changes in kinetics of spontaneous events, resulting in faster, briefer mGPSCs at E18 compared with E14.

\section{Developmental changes in membrane responses in I-clamp} A hallmark of NM neurons as well as other time encoding neurons in the auditory pathway is high-pass filtering (Oertel, 1983; Reyes et al., 1994). When depolarized with a long-duration current step, NM neurons generally fire only a single AP at the onset of the current step. This spike is followed by a stable membrane potential and no additional APs occur until after the offset of the stimulus (Reyes et al., 1994). We studied the occurrence of this phenomenon in current-clamped NM neurons from E10-E18 
A

E10

E12
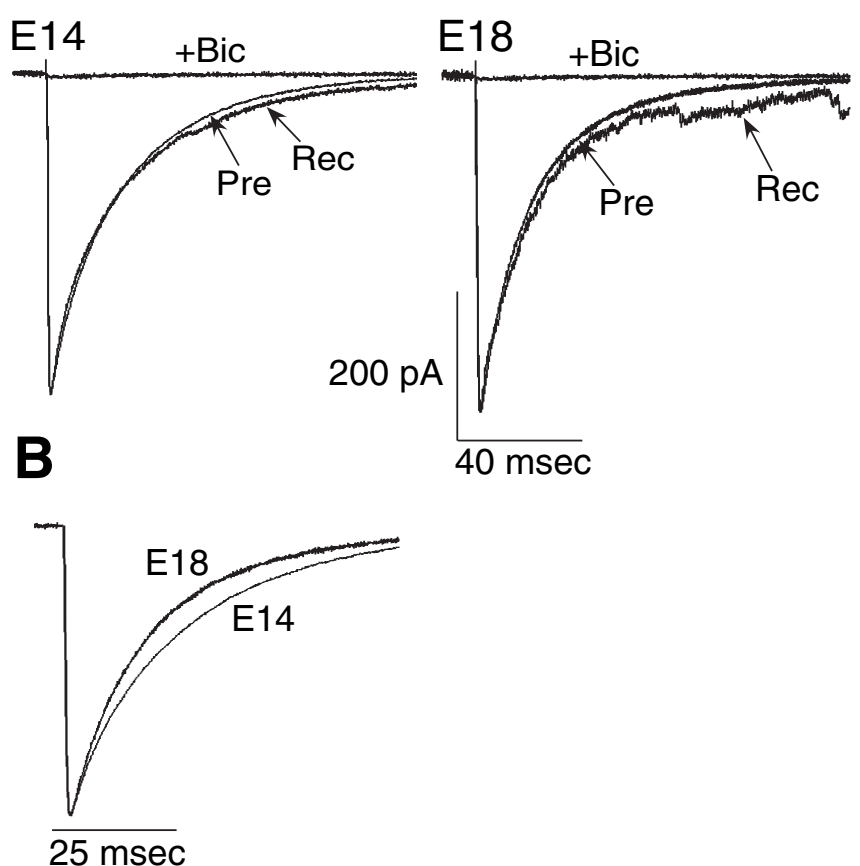

Figure 3. GPSC decay kinetics become faster across development. All GPSCS were recorded at a holding potential of $-60 \mathrm{mV}$ during bath application of DNQX and AP5. A, Averaged GPSC traces from representative E10, E12, E14, and E18 neurons. GPSCs were first recorded at E14. Preliminary (Pre) GPSCs were completely blocked with bath application of Bic (+Bic), but recovered to normal levels after washout (Rec). Stimulus artifacts have been removed for clarity. B, E18 GPSCs exhibit faster decay kinetics than E14 when current amplitude-normalized GPSCS are overlaid. The overlaid traces illustrate the faster decay kinetics in more mature neurons.

using depolarizing current steps ranging from 50 to $500 \mathrm{pA}$ (150 ms duration) in 50 pA steps (Fig. 4A). Representative responses of neurons at each age are illustrated in Figure $4 B$. Voltage-current $(V-I)$ relations, in which the steady state membrane potential, measured as the average potential over the last $5 \mathrm{~ms}$ of the current step (Fig. $4 \mathrm{~A}$, asterisk), is plotted as a function of injected current are shown in Figure $4 C$ for each age.

In general, NM neurons at E10 do not maintain clamped membrane potentials during long-duration depolarizing current steps as well as older NM neurons do, often firing multiple APs clustered near the beginning of the stimulus (Fig. $4 B$ ). It should be noted, however, that these neurons do not fire APs throughout the current step, as do many other types of neurons (Reyes et al., 1994). Only a subset of E12 neurons (four of nine) fired a second spike in response to large current injections. By E14, only one of 13 neurons sampled fired a second spike during the larger current steps, and in no case did E18 neurons fire multiple spikes.

Mature NM neurons have low input resistances compared with younger neurons, indicated by the flattened $V-I$ curve (Fig. $4 C)$. The mean steady-state membrane potentials of E10 and E12 NM neurons in response to a step as small as $50 \mathrm{pA}$ are significantly depolarized compared with E18 neurons $(p<0.05$, ANOVA, Scheffe post hoc comparison). Responses to all larger current steps, $100 \mathrm{pA}$ and up, of neurons at E10, E12, and E14 are also significantly depolarized compared with E18 $(p<0.05$ for
A

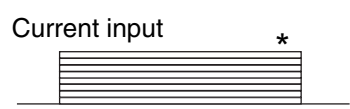

B

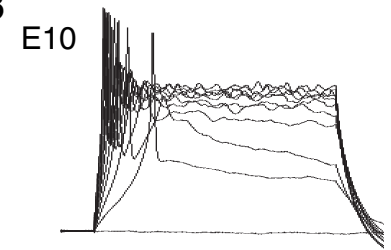

E12
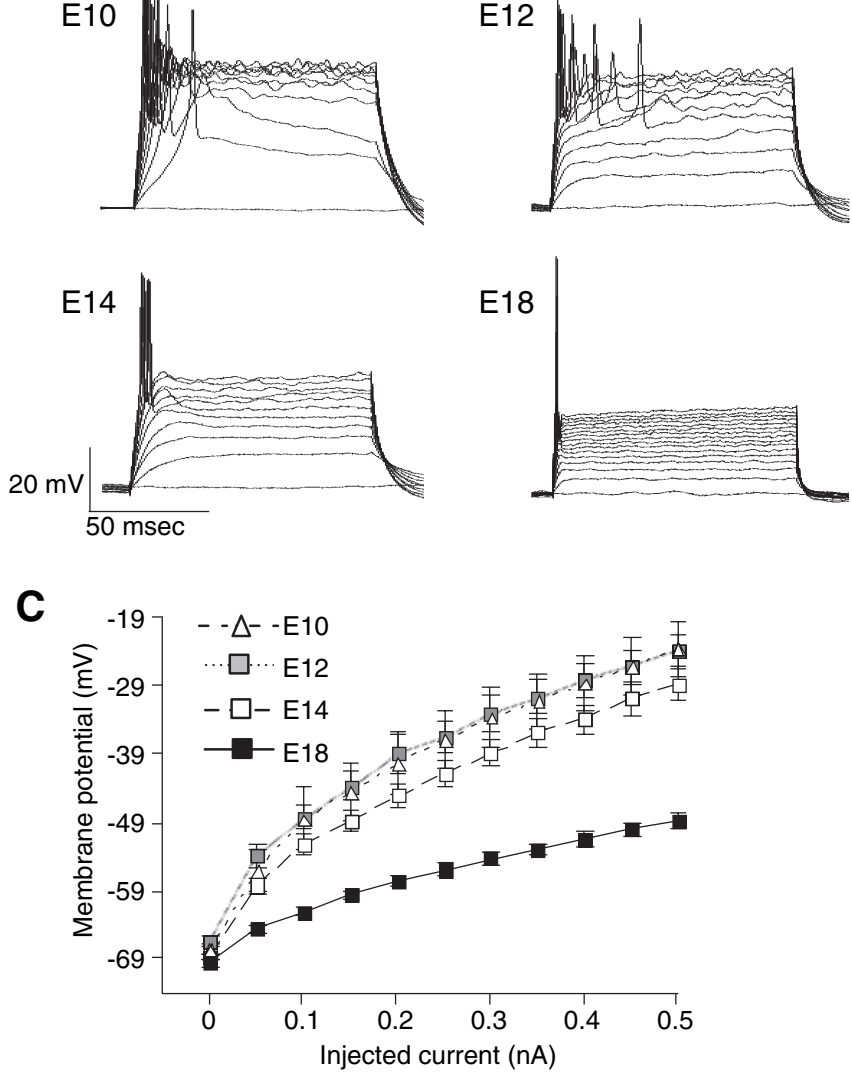

Figure 4. Input resistance is reduced and single AP firing is more prevalent across development. These experiments were performed using current clamp in the presence of bath-applied DNQX, AP5, and Bic. A, Depolarizing currents steps ranging from 0 to $750 \mathrm{pA}$ in $50 \mathrm{pA}$ steps. The asterisk marks the time at which steady-state membrane potential was measured. $\boldsymbol{B}$, Example responses from representative E10, E12, E14, and E18 neurons. The E10 and E12 neurons each exhibit multiple spikes when stimulated with the largest current steps; E14 and E18 neurons do not. In older neurons the spikes occur and decay much faster. C, Voltage-current relations consist of steady-state membrane potential (averaged across the last $5 \mathrm{~ms}$ of the currents step) plotted as a function of injected current. The flattened $V-I$ function of E18 neurons (black squares) is indicative of lower input resistance and larger $\mathrm{K}^{+}$conductances compared with other ages. The change in membrane potential is significantly less in E18 neurons at all current amplitudes $(p<0.05)$. Error bars for this and all other figures represent SEM.

100 pA step; $p<0.01$ for 150 pA step; $p<0.001$ for all larger steps). These analyses revealed no significant differences between the membrane responses of E10, E12, and E14 neurons to depolarizing current steps.

Pharmacological studies have attributed the low input resistance and, thus, the flattened $V-I$ relations of NM neurons in part to the high expression of the family of low-voltage-activated $\mathrm{K}^{+}$ channels in the membrane (Reyes et al., 1994; Rathouz and Trussell, 1998). Bath application of DTX-I, a specific blocker of channels containing at least one Kv1.1-, 1.2-, or 1.6-type subunit, disrupts the high-pass filtering exhibited by NM neurons (Rathouz and Trussell, 1998; Slee et al., 2005). Our data support these previous findings; in the presence of DTX-I, rheobase decreased such that the smallest depolarizing current step often elicited APs, and neurons reliably fired multiple spikes during current steps (data not shown). 


\section{Development of $\mathrm{K}^{+}$conductance}

LVA outward currents are large in NM and neurons of many nuclei of the lower auditory system (for review, see Trussell, 1997). $\mathrm{K}^{+}$channels of the Kv1 family, encoded by the KNCA gene family, carry much of this current. We used voltage-clamp protocols to measure $\mathrm{LVA} \mathrm{K}^{+}$currents activated between resting membrane potential and AP threshold (i.e., between -70 and $-45 \mathrm{mV}$ ) in NM neurons across development. Neurons were perfused with a low-Ca ${ }^{2+}(0.5 \mathrm{~mm})$ ACSF that included blockers of voltage-activated $\mathrm{Na}^{+}$channels (TTX) and $I_{\mathrm{h}}(\mathrm{ZD}-7288)$ as well as DNQX, AP5, and Bic. Figure $5 A$ illustrates the voltage step protocol used, in which neurons were initially held at a potential close to rest, $-70 \mathrm{mV}$. Membrane potential was stepped to test potentials ranging from -75 to $-45 \mathrm{mV}$, in $5 \mathrm{mV}$ steps of $200 \mathrm{~ms}$ duration, returning to the holding potential of $-70 \mathrm{mV}$ for 300 ms between steps. Representative examples of the $\mathrm{K}^{+}$currents activated by this protocol from E14 and E18 NM neurons are shown in Figure $5 B$. Current-voltage $(I-V)$ curves, produced by plotting the average peak current (occurring 2-5 ms after onset of the voltage step) against the test potential are displayed in Figure $5 C$. Leak subtraction methods were applied to $I-V$ curves, using the current recorded during the hyperpolarizing step from -70 to $-75 \mathrm{mV}$ as a baseline.

In E18 neurons, all depolarizing voltage steps $(\geq-65 \mathrm{mV})$ activate significant $\mathrm{K}^{+}$currents and E14 neurons exhibit significant currents to voltage steps $\geq-60 \mathrm{mV}$ ( $p<0.05, t$ tests). Comparison of $\mathrm{K}^{+}$currents and $I-V$ curves across age reveals a large increase in $\mathrm{K}^{+}$-current magnitude with age. $\mathrm{K}^{+}$currents were significantly larger in E18 neurons compared with E14 neurons during all depolarizing voltage steps ( $p<0.05, t$ tests).

To determine the $\mathrm{K}^{+}$channels involved in the $\mathrm{LVA} \mathrm{K}^{+}$currents in NM neurons, DTX-I was bath applied for a minimum of 4 min before a second recording of $\mathrm{K}^{+}$currents. $I-V$ curves recorded during DTX-I application show large reductions in $\mathrm{K}^{+}$ currents (Fig. 5C). DTX-I significantly suppressed $\mathrm{K}^{+}$-current magnitudes during all voltage steps in E14 and E18 neurons $(p<$ $0.05, t$ tests). In E18 neurons, $69 \%( \pm 15)$ of the peak $\mathrm{K}^{+}$current was blocked by DTX-I during a step in membrane potential from -70 to $-60 \mathrm{mV}$. The decrement was similar at E14, and the percentage block at other depolarizing steps was similar. Previous reports of voltage-clamp studies of the medial nucleus of the trapezoid body (MNTB) describe tissue penetration problems for dendrotoxins into slices, with complete Kv1 current block occurring only after incubation in dendrotoxins for tens of minutes (Gittelman and Tempel, 2006). Our experiments were not this long lasting; thus, we expect that unaffected Kv1 channels carried a portion of the residual current. The residual "DTX-insensitive" currents are similar to DTX-insensitive currents recorded in mouse MNTB and to $\mathrm{LVA} \mathrm{K}^{+}$currents recorded in MNTB neurons of Kv1.1 knockout mice (Brew et al., 2003). Overall, these data indicate a major increase in the voltage-dependent currents, particularly Kv1 currents, which would be activated by depolarizing GPSPs in NM neurons between E14 and E18.

\section{Development of AP inhibition by depolarizing GPSPs}

Depolarizing GPSPs are potently inhibitory in mature (E18) NM neurons (Monsivais and Rubel, 2001). We compared the efficacy of depolarizing GPSPs in spike inhibition in E14 NM neurons, when synaptic responses and $\mathrm{LVA} \mathrm{K}^{+}$conductances are immature, with the efficacy of depolarizing GPSPs in spike inhibition in mature E18 neurons. The protocol for these experiments is illustrated in Figure $6 \mathrm{~A}$. Neurons were stimulated with two depolarizing current pulses, each $2 \mathrm{~ms}$ in duration, with an inter-

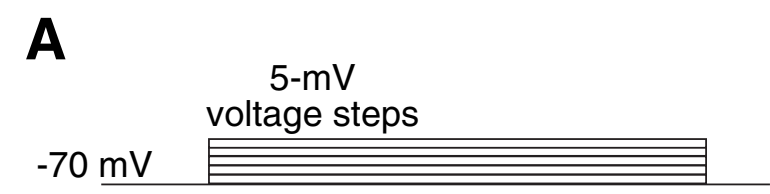

B
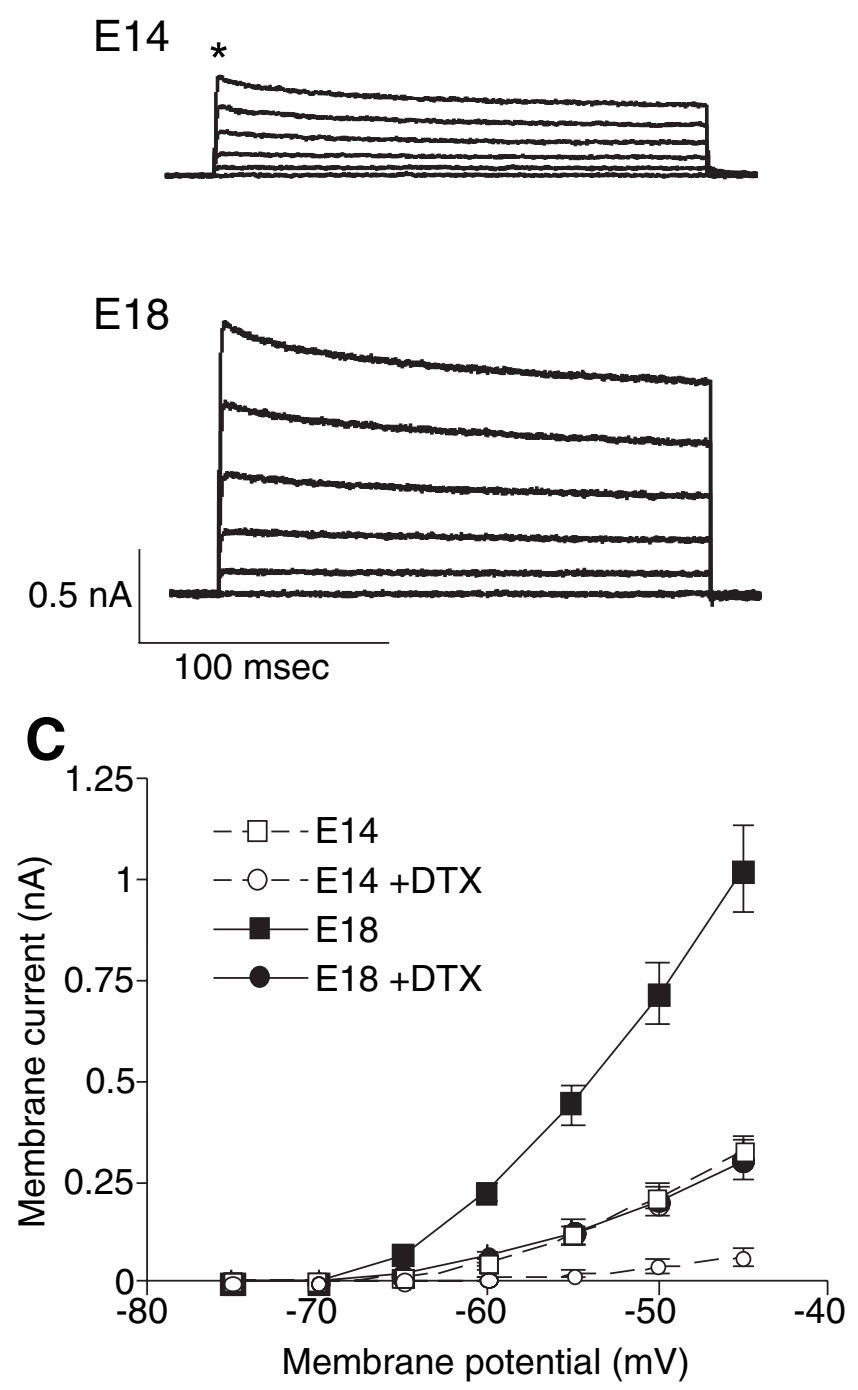

Figure 5. $\mathrm{LVAK}^{+}$currents increase with age in NM neurons. These experiments were conducted with DNQX, AP5, Bic, TTX, QX-314, and low $\mathrm{Ca}^{2+}$ present in the bath. $\boldsymbol{A}$, Protocol in which neurons were held at $-70 \mathrm{mV}$ and stepped from -75 to $-45 \mathrm{mV}$ in $5 \mathrm{mV}$ steps. $\boldsymbol{B}$, Representative $\mathrm{K}^{+}$currents recorded from E14 and E18 neurons. The amplitude of these currents is much greater in mature E18 neurons. The asterisk marks the point at which the peak current was measured. $C$, Current-voltage curves consist of peak current plotted against test potential. E18 ( $n=7$; filled squares) neurons showed significantly greater current amplitudes than did E14 neurons ( $n=7$; open symbols) in response to all depolarizing current steps ( $p<$ 0.05). DTX-I (circles) greatly reduces these currents at both ages. These functions were leak subtracted using response to the hyperpolarizing step from -70 to $-75 \mathrm{mV}$ as a baseline. A large portion of the LVA K ${ }^{+}$current was blocked in both E14 $(63 \% \pm 18 ; n=6)$ and E18 neurons $(69 \% \pm 6 ; n=7)$, indicating a major role for Kv1-type channels in the LVA K ${ }^{+}$ currents activated by depolarizing GPSPS.

stimulus interval of $100 \mathrm{~ms}$. Current pulse amplitude was increased from 0.05 to $1.0 \mathrm{nA}$ in $50 \mathrm{pA}$ steps. Ten milliseconds before the second current pulse, a GPSP was evoked with an extracellular stimulating electrode (in the presence of AP5 and DNQX) as described above. Mean GPSP amplitudes were similar 
A

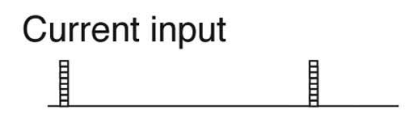

Synaptic input
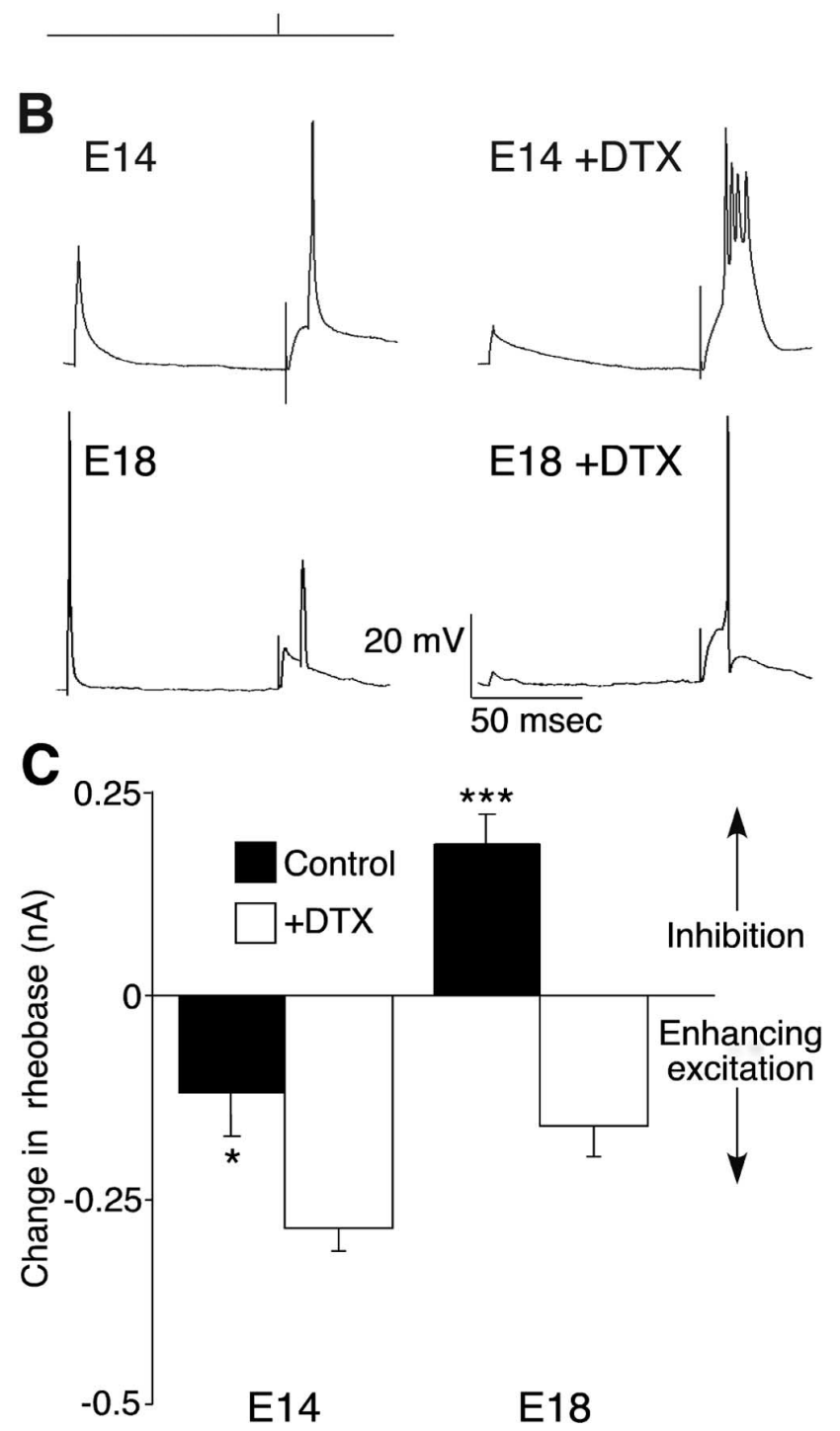

Figure 6. GABAergic inputs enhance excitability at E14 but are inhibitory at E18 in NM neurons. $\boldsymbol{A}$, Neurons held in whole-cell current clamp were stimulated with depolarizing current pulses of variable amplitude. GABAergic synaptic stimulation was performed with a bipolar electrode in the presence of bath-applied DNQX and AP5. $\boldsymbol{B}$, Under control conditions, GPSPS summated with current pulses in E14 neurons to induce spiking, but shunted current pulses at E18 to prevent spiking (left column; stimulus artifacts reduced for clarity). Addition of DTX-I to the bath (right column) induced summation between GPSP and current pulses in neurons of both ages. C, Rheobase during a GPSP was subtracted from the control condition for E14 $(n=6)$ and E18 $(n=7)$ neurons. All E14 neurons $(n=6)$ exhibit negative changes in rheobase, indicating enhancement of excitability. This effect was significantly enhanced by addition of DTX-I ( $\left.{ }^{*} p<0.05\right)$. At E18, positive values for change in rheobase indicate shunting and inhibition during GPSPS. DTX-I application reversed this effect, making GPSPs excitatory ${ }^{* * *} p<$ 0.001). Upward and downward arrows indicating inhibition and enhancement of excitation are for clarity.

between ages E14 and $\mathrm{E} 18(\mathrm{E} 14,7.4 \pm 4.0 \mathrm{mV}, n=5 ; \mathrm{E} 18,8.4 \pm$ $4.5 \mathrm{mV}, n=5$; not significant by $t$ test). The first current pulse served to measure control rheobase, defined as the current pulse amplitude that evoked APs $100 \%$ of the time. An AP was defined to have occurred when the upward deflection of the membrane potential contained an upward inflection point. This control measure was then subtracted from rheobase recorded during GPSPs. A positive change in rheobase during a GPSP indicates inhibition of the current pulse by the GPSP whereas a negative change in rheobase indicates summation between the two inputs and enhancement of excitability by the GPSP. Neurons were tested with this protocol multiple times to ensure reproducibility.

Figure $6 B$ illustrates responses from representative E14 and E18 neurons to this protocol. At E14, the current pulse that elicits a subthreshold depolarization sums with the GPSP to evoke a spike. This indicates that GPSPs enhance the excitability of neurons at this age. The opposite can be seen at E18, where the depolarizing GPSP blocked AP generation by a current pulse that was suprathreshold under control conditions. This indicates shunting inhibition of the current pulse by the GPSP (Monsivais and Rubel, 2001). Bath application of DTX-I converted GABAergic inhibition to excitation. Monsivais and Rubel (2001) reported spikes evoked by miniature GPSPs during DTX-I application. We also observed this phenomenon in all E18 neurons and in eight of nine E14 neurons (data not shown). DTX-I hyperpolarized AP voltage threshold to potentials close, or hyperpolarized to $E_{\mathrm{GABA}}$ (Table 1). In E18 neurons, DTX-I also resulted in summation between subthreshold GPSPs and current pulses to evoke spikes, as in E14 neurons both in control and DTX-I conditions. Group data are presented in Figure 6C. In E14 neurons, rheobase was changed by an average of $-0.12 \mathrm{nA}( \pm 0.15)$ during a GPSP $(p<$ $0.05, t$ test; $n=9)$. DTX-I application enhanced this effect, with spikes evoked by current pulses that were $-0.28 \mathrm{nA}( \pm 0.09)$ smaller during GPSPs than control. The changes in rheobase during a GPSP in the presence of DTX-I were significant, indicating enhanced excitation by GPSPs when Kv1 channels were blocked $(p<0.05, t$ test). By E18, there was a dramatic change in how NM neurons responded to GABAergic inputs. Rheobase during a GPSP was greater than control by $0.19 \mathrm{nA}( \pm 0.11)$, indicating significant inhibition ( $p<0.05, t$ test; $n=7)$. In contrast, during DTX-I application, rheobase was decreased by $-0.16 \mathrm{nA}( \pm 0.11)$ during a GPSP relative to the control measurement, significantly changed from the non-DTX-I value $(p<0.001, t$ test $)$. It is important to note that the control measurements are also made in the presence of DTX-I, thus, eliminating the potentially confounding factor of the change in AP voltage threshold caused by DTX-I. Our results indicate that DTX-I-sensitive Kv1 channels were necessary for shunting inhibition, with a reversion from inhibition to excitation by GPSPs when these channels were blocked in E18 neurons.

Previous studies from this laboratory and others have examined the effects of trains of GABAergic input to NM neurons by stimulating superior olivary nucleus (SON) axons at high frequencies (Lu and Trussell, 2000; Monsivais et al., 2000). Trains of GPSPs temporally sum, inducing a steady-state depolarized potential during which APs are strongly inhibited. LVA K ${ }^{+}$conductances are activated tonically during these depolarizations. As stimulation frequency is increased, GABA release becomes asynchronous, extending the time course of potential inhibition beyond the cessation of the stimulus train (Lu and Trussell, 2000). We tested the power of these GABA-induced steady-state depolarized potentials to inhibit spiking in E14 and E18 NM neurons. The protocol for these experiments is illustrated in Figure $7 A$. We stimulated NM neurons with a train of 25 excitatory current pulses ( 2 ms duration), applied with an interstimulus interval of $20 \mathrm{~ms}$ (frequency, $50 \mathrm{~Hz}$ ), increasing in amplitude from 0 to 1.5 $\mathrm{nA}$ in $100 \mathrm{pA}$ steps. The first two current pulses served as controls 


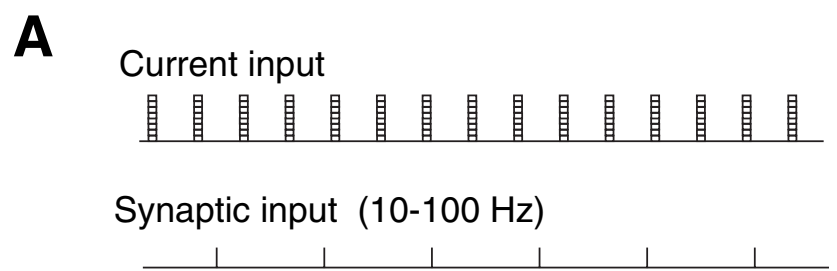

B

E14

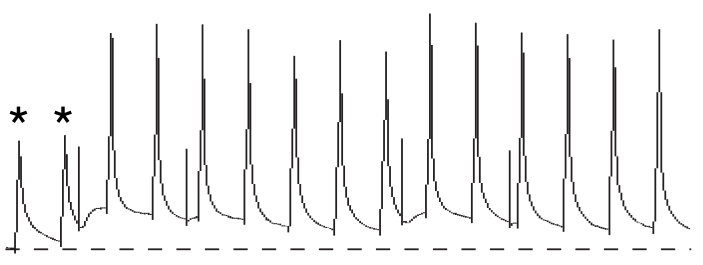

E18
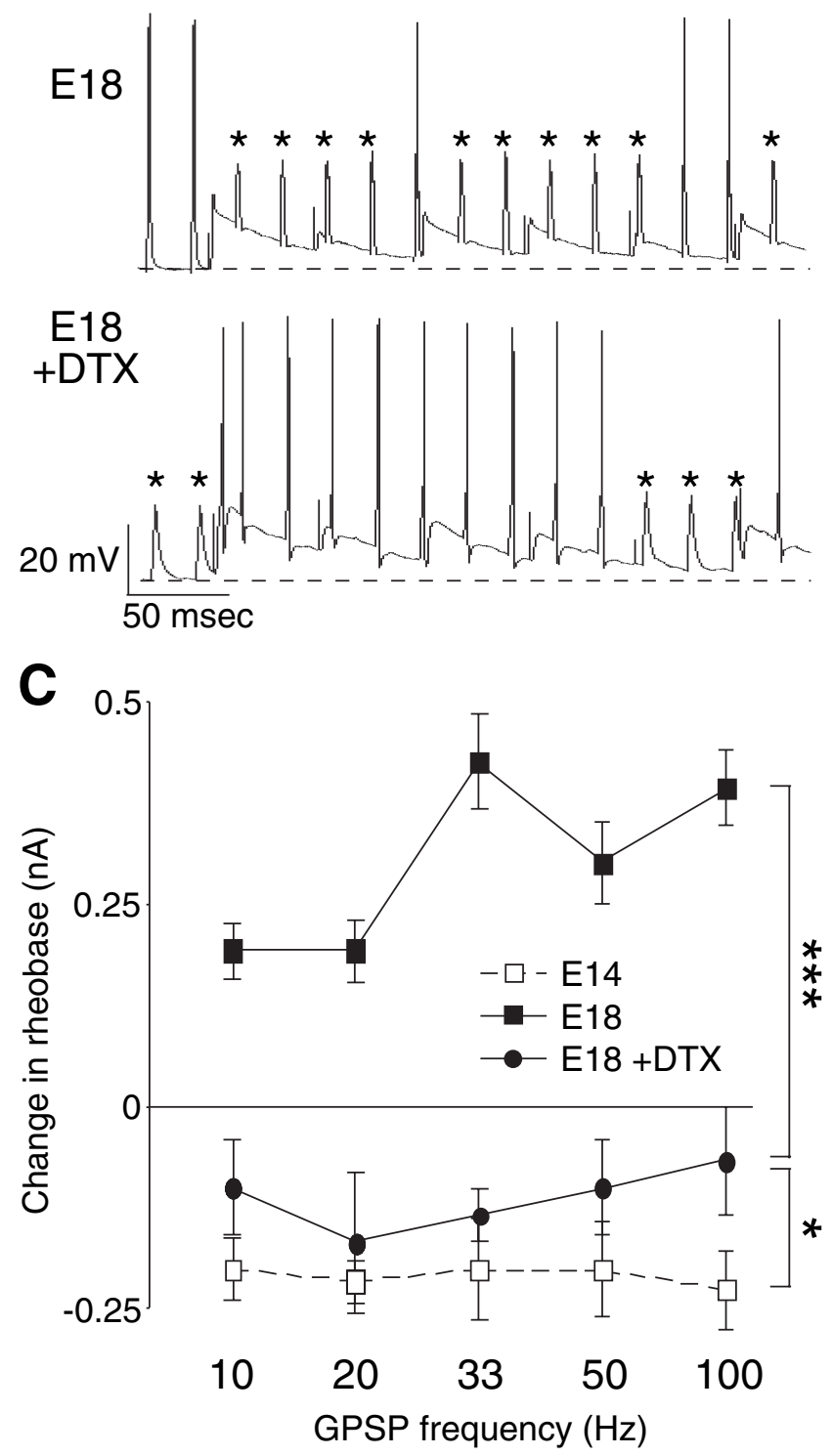

Figure 7. GPSP trains enhance excitability at E14 and inhibitory at E18 in NM neurons. $\boldsymbol{A}$, Neurons held in whole-cell current clamp were stimulated with trains of depolarizing current pulses at $50 \mathrm{~Hz}$. Simultaneously, trains of GPSPs were stimulated extracellularly at frequencies ranging from 10 to $100 \mathrm{~Hz}$ ( $20 \mathrm{~Hz}$ shown in $\boldsymbol{B}$ ) in the presence of DQX and AP5. $\boldsymbol{B}$, Summation between the GPSP-induced steady-state depolarization and current pulses causes an E14 neuron (top trace) to fire in response to current pulses that stimulate only subthreshold responses (marked with asterisks) under control conditions. The opposite is true in an E18 neuron (middle trace), in which firing is blocked during most of the GPSP train except when the membrane to measure the control rheobase. After the second current pulse, SON fibers were stimulated at frequencies ranging from $10-100$ $\mathrm{Hz}$ (Fig. $7 A, B, 50 \mathrm{~Hz}$ ) in the presence of AP5 and DNQX. The change in rheobase during the stimulus train was calculated as above for the 10th current pulse. This pulse was chosen because it occurred during the steady-state depolarized potential for all stimulation frequencies.

Figure $7 B$ shows the responses of representative E14 and E18 neurons to current pulse trains during $20 \mathrm{~Hz}$ GABAergic synaptic stimulation. The bottom trace illustrates the response of an E18 neuron during bath application of DTX-I. Asterisks mark current pulses that failed to evoke a spike. In E14 neurons, current pulses that were subthreshold under control conditions evoked spikes during the GABAergic steady state potential. This indicates summation and an enhancement of excitability by GABA. E18 neurons showed the opposite effect, in which suprathreshold current pulses were shunted and spikes were inhibited during the GABAergic train. The spikes that were generated tended to occur when the membrane depolarization decayed closer to the resting membrane potential (marked by dashed line). Spike inhibition by GPSP trains at E18 was converted to excitation with bath application of DTX-I; subthreshold current pulses evoked APs during the GPSP train (Fig. 7B), as did GPSPs alone (data not shown), as in the E14 example. Group data for this experiment are shown in Figure 7C. In E14 neurons, rheobase changed by at least $-0.2 \mathrm{nA}$ for each GPSP stimulus frequency used, indicating significant summation regardless of input frequency $(p<0.05, t$ test). An ANOVA indicated no significant difference in the change in rheobase across stimulus frequencies for this age group. E18 neurons showed positive shifts in rheobase during GPSP trains, indicating inhibition at all stimulus frequencies. Increasing stimulus frequency had a significant effect on the strength of this inhibition, with 33,50 , and $100 \mathrm{~Hz}$ showing significantly greater increases in rheobase than the lower frequencies of 10 and $20 \mathrm{~Hz}(p<0.05$, ANOVA, Scheffe post hoc comparisons). Bath application of DTX-I significantly decreased rheobase during GPSP trains compared with control measures $(p<0.001$, ANOVA). However, rheobase reduction during GPSP trains in E18 neurons during DTX-I application was significantly less in E14 neurons during control conditions $(p<$ 0.05 , ANOVA). These data, in addition to those of the previous experiment, indicate that the maturation of Kvl currents was necessary for GABA to functionally inhibit spiking in mature NM neurons. We did not observe changes in AP voltage threshold across the AP burst evoked by these experiments. Such changes could potentially occur across longer bursts, but did not affect the data presented here.

To separate the contribution of the voltage-dependent $\mathrm{K}^{+}$ conductance to shunting inhibition from that of the synaptic conductance, we designed an experiment using depolarizing current steps to activate only the voltage-dependent component of shunting inhibition. Monsivais et al. (2000) showed that depolarizing current steps did not have the same inhibitory effect that trains of GABAergic GPSPs had. We expanded on this work using a greater range of stimuli and compared differences in summa-

approaches rest (marked by dashed line). Excitation is enhanced in E18 neurons exposed to DTX (bottom trace), similar to E14. C, Decreases in rheobase during GPSP trains indicate enhanced excitability in E14 $(n=9)$ neurons and E18 + DTX $(n=3)$, whereas increases in rheobase at E18 $(n=7)$ indicate spike inhibition. This pattern is observed at all GPSP frequencies. DTX significantly shifted inhibition to excitation in E18 neurons ( $\left.{ }^{* *} p<0.001\right)$, but enhanced excitability significantly less than GPSPs at E14 $\left({ }^{*} p<0.05\right)$. 
A Current input

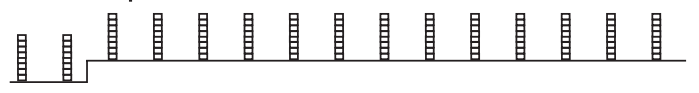

B $\quad$ 14 $(I=0.1 \mathrm{nA})$
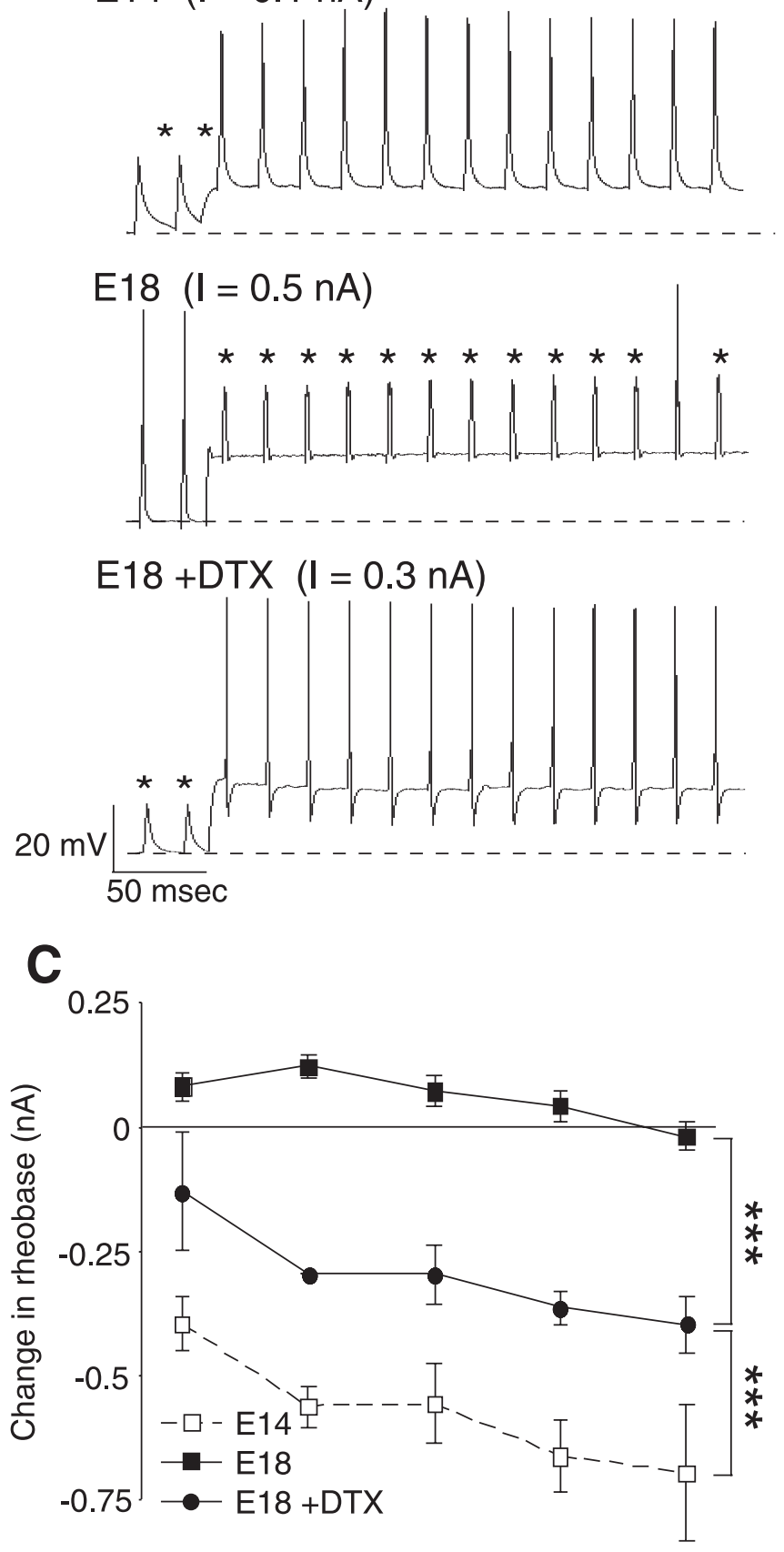

\section{$\begin{array}{lllll}0.1 & 0.2 & 0.3 & 0.4 & 0.5\end{array}$ \\ Current step amplitude (nA)}

Figure 8. Subthreshold depolarizing current injections enhance excitability at E14 and inhibitory at E18 in NM neurons. $\boldsymbol{A}$, Neurons held in whole-cell current clamp were stimulated with $50 \mathrm{~Hz}$ trains of depolarizing current pulses. After the second control pulse a depolarizing current step, ranging from 0.1 to $0.5 \mathrm{nA}$ was activated. $\boldsymbol{B}$, A representative E14 neuron (top trace) that displayed subthreshold responses to control current pulses (marked with asterisks) fired spikes in response to the same current pulses after the onset of the current step. The middle trace illustrates a representative E18 neuron in which spikes were fired during control conditions but were shunted to subthreshold (asterisks) during the current step. An E18 neuron exposed to DTX (bottom trace) fired repetitively to current pulses during the current step. E14 tion or inhibition during depolarizing current steps in NM neurons at E14 and E18. The protocol for these experiments is illustrated in Figure $8 \mathrm{~A}$. APs were evoked using a train of current pulses, as in the previous experiment. After the second control current pulse, a long-duration (500 ms) depolarizing current step was activated such that subsequent current pulses were added on top of this step. Trials were separated by a period of $150 \mathrm{~ms}$ after the offset of the depolarizing current step. Separate recordings were made using long-duration steps ranging in amplitude from 0.1 to $0.5 \mathrm{nA}$, in $0.1 \mathrm{nA}$ steps.

Responses of representative E14 and E18 neurons, and E18 neurons exposed to bath applied DTX-I are shown in Figure $8 B$. Equal current steps induced depolarizations of differing amplitudes in neurons of different ages (Fig. 4). Thus, the examples illustrated in Figure $8 B$ exhibit equivalent depolarizations but used different amplitude current steps. At E14, the current pulses that elicited only subthreshold depolarizations under control conditions evoked spikes when the neuron was depolarized. The opposite was true at E18, where suprathreshold current pulses failed to elicit APs when the neuron was depolarized. The response of the neuron to the depolarizing current step reverted to the immature, excitatory form during application of DTX-I. In the presence of DTX-I, APs were evoked during the current step by current pulses that were subthreshold under control conditions. Group data for these experiments are shown in Figure $8 C$. Increasing the depolarizing current step size systematically decreased rheobase in E14 neurons. The effect of current step size was significant ( $p<0.05$, ANOVA). Thus, immature NM neurons became more excitable as the membrane was depolarized to greater degrees. In E18 neurons, however, rheobase increased during all depolarizing current steps. Current pulses were shunted and excitability reduced during depolarization. E18 neurons bathed in DTX-I were significantly more excitable than under control conditions, but significantly less excitable than E14 neurons $(p<0.001$, ANOVA). This indicates that the maturation of Kvl currents was sufficient to result in shunting of excitation in mature neurons, even in the absence of the synaptic conductance. As with the above experiments using synaptic stimuli, we did not observe changes in AP voltage threshold during the short bursts of activity evoked by some of the stimuli. Although such changes could occur in response to longer stimuli, they are not apparent in our data.

Previous experiments by Monsivais et al. (2000, 2001) suggest that the $\mathrm{GABA}_{\mathrm{A}} \mathrm{R}$ synaptic conductance is the dominant component of shunting inhibition in NM. In this report, stimuli included both trains of GABAergic synaptic potentials that activate both synaptic and voltage-activated membrane conductances (Fig. 7), and depolarizing current steps that activate only the voltage-activated membrane conductances (Fig. 8). We compared the results from the two previously described experiments to understand the roles these two conductances play in shunting inhibition in NM neurons. Figure 9 illustrates the changes in rheobase during synaptic trains and current steps at ages E14 and

$\leftarrow$

and E18 examples that exhibited equivalent depolarization in response to the current step were selected for this comparison. I indicates the current step size for each trace. C, Depolarizing current steps of all amplitudes decreased rheobase (were excitatory) in E14 neurons ( $n=9)$. The enhancement of excitability increased significantly as current step size increased. Spikes were inhibited (rheobase increased) during all but the largest depolarizing current steps in E18 neurons $(n=7)$. Exposure to DTX converted inhibition to excitation in E18 neurons $(n=3)$, significantly decreasing rheobase $\left(^{* * *} p<0.001\right)$, while enhancing excitability less than in E14 neurons $\left({ }^{* *} p<0.001\right)$. 


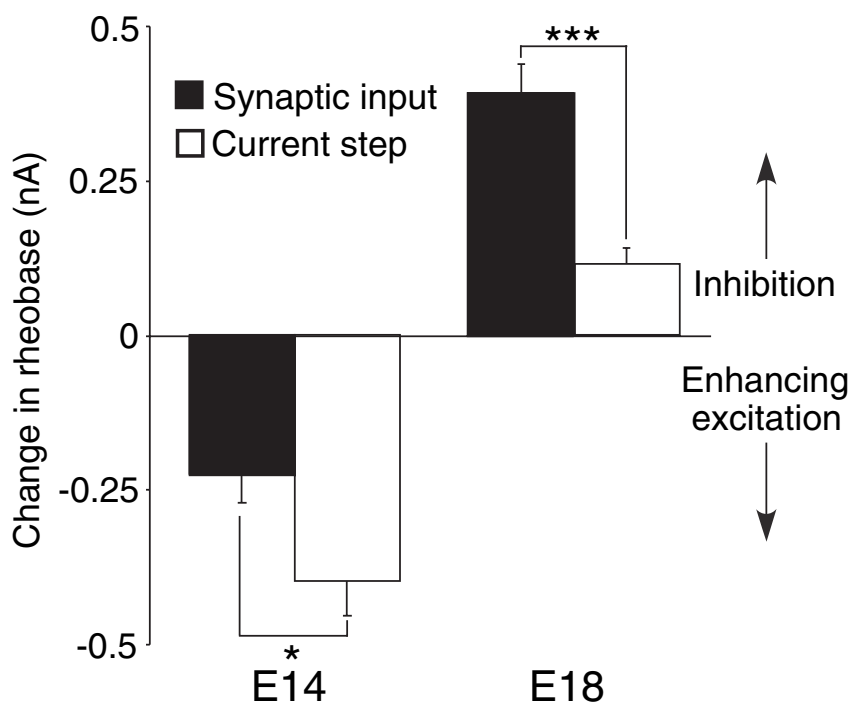

Figure 9. GPSPs induce the greatest inhibition in E18 neurons. Inhibition/enhancement of excitability by GPSP trains and current step that induce equivalent depolarizations ( $\sim 10 \mathrm{mV}$ ) are compared. At E14, enhancement of excitation is significantly greater during current steps than GPSP trains because of the lack of the shunting synaptic conductance. At E18, inhibition is strongest during GPSP trains because of shunting by both synaptic and voltage-activated conductances. ${ }^{*} p<0.05$; ${ }^{* *} p<0.001$.

E18. For this analysis, the experimental conditions of synaptic input frequency and current step amplitude that induce equivalent depolarizations $(\sim 10 \mathrm{mV})$ were selected. For E14 neurons, these were $100 \mathrm{~Hz}$ and $0.1 \mathrm{nA}$, whereas for E18 neurons these were $100 \mathrm{~Hz}$ and $0.3 \mathrm{nA}$. Both trains of GPSPs and depolarizing current steps enhance excitability (decrease rheobase) in immature E14 neurons. However, the additional conductance provided by activated $\mathrm{GABA}_{\mathrm{A}}$ Rs leads to a shunting of some of this excitation, resulting in significantly smaller changes in rheobase during the GPSP train stimuli than during the depolarizing current step ( $p<0.05, t$ test). In the more mature E18 neurons, GABAergic inputs and depolarizing current steps are both inhibitory (increase rheobase). The additional conductance of the $\mathrm{GABA}_{\mathrm{A}}$ Rs during GPSP trains resulted in greater shunting, leading to significantly larger changes in rheobase, than during depolarizing current steps ( $p<0.005, t$ test). This comparison illustrates that in immature E14 neurons the enhancement of excitability as neurons were depolarized toward AP threshold dominated the shunting effect of both the synaptic and small voltage-dependent $\mathrm{K}^{+}$conductances. In more mature E18 neurons, the large voltage-dependent $\mathrm{K}^{+}$conductance alone, activated by depolarization, shunted excitation. However, the combination of synaptic and voltage-dependent $\mathrm{K}^{+}$conductances in E18 neurons made shunting inhibition even more powerful.

\section{Discussion}

In this report, we provide a detailed developmental analysis of the neuronal properties that support depolarizing GABAergic inhibition in nucleus magnocellularis (NM) neurons. Our major finding is that these neurons undergo a dramatic switch in their response to GABA despite relative stability in the reversal potential of GPSPs. At younger ages (E14), depolarizing GPSPs enhance excitability of NM neurons whereas at older ages (E18), depolarizing GPSPs strongly inhibit spiking by shunting and spike threshold accommodation. The onset of inhibition correlates with specific developmental changes in the voltage-activated membrane properties of these neurons. Depolarizing GPSPs are transformed from summating to shunting by increases in LVA $\mathrm{K}^{+}$currents carried mostly by Kv1-type channels. We also demonstrate in a functional assay that application of DTX-I, a specific Kv1 current blocker, restores the immature "phenotype" with GABA enhancing excitability. These results provide a novel mechanism by which the developmental sign inversion of GABAergic signaling is achieved.

Many immature neurons, or newly generated neurons in adult CNS, maintain relatively high intracellular $\mathrm{Cl}^{-}$concentrations, rendering GABAergic or glycinergic synaptic potentials depolarizing and excitatory (Owens et al., 1996; Ehrlich et al., 1999; Kakazu et al., 1999; Balakrishnan et al., 2003; Ge et al., 2006). Most neurons undergo a developmental switch involving changes in $\mathrm{Cl}^{-}$transporter expression and $\mathrm{E}_{\mathrm{Cl}^{-}}$such that the polarity of GABAergic potentials becomes hyperpolarizing and inhibitory (Rivera et al., 1999). Some hippocampal inhibitory interneurons maintain a shunting inhibitory response to GABA throughout development (Banke and McBain, 2006). However, GABAergic responses by NM neurons are unusual in that they remain depolarizing into maturity (Hyson et al., 1995). We demonstrate that NM neurons undergo a developmental conversion in GABA responses while $E_{\mathrm{Cl}^{-}}$and, thus, GPSP polarity remains depolarized compared with resting membrane potential.

Previous models of depolarizing inhibition have examined the individual and combined roles of synaptic and voltagedependent conductances in spike inhibition. In the case of presynaptic depolarizing inhibition, GABAergic synaptic conductances may provide the sole source of shunting, reducing $\mathrm{Ca}^{2+}$ influx and transmitter release (Graham and Redman, 1994). Other models show subthreshold GABAergic depolarizations diminishing or blocking spiking by inactivating voltage-activated $\mathrm{Na}^{+}$channels (Eccles et al., 1961). Activation of voltagedependent outward currents adds to the shunting effect of GABAergic synaptic conductances and enhances inhibition of spiking (Edwards, 1990a,b).

Our data are consistent with studies of developmental changes in kinetics and frequency of GABAergic events. Three potential mechanisms could lead to such changes. First, NM neurons may undergo developmental changes in $\mathrm{GABA}_{\mathrm{A}} \mathrm{R}$ subunit expression, as do other neurons exhibiting similar changes in $G_{A B A} R$ currents (Okada et al., 2000). Second, developmental changes in presynaptic release machinery that alter vesicle synchrony, as seen in the mammalian brainstem (Magnusson et al. 2005), could account for the changes in GPSC properties described here. Third, a reduction in dendritic filtering caused by developmental retraction of complex dendritic arbors between E10 and E18 (Jhaveri and Morest, 1982) could give similar results. Our data do not differentiate between these possible mechanisms. The changes in synaptic conductance we report are not consistent with the substantial increase in membrane conductance required for the switch to shunting inhibition. This further implies that changes in the voltage-dependent properties of the neurons are responsible for the emergence of shunting inhibition.

Strong outward $\mathrm{K}^{+}$currents are expressed in NM and other auditory nuclei. At resting membrane potential, a population of the LVA K ${ }^{+}$channels is open. Subthreshold (and suprathreshold) depolarizations from resting membrane potential induced by either EPSPs and depolarizing GPSPs results in further activation of these channels. We describe a large increase in the magnitude of these currents between E14 and E18 in NM. Using RTPCR, Duzhyy et al. (2004) showed increases in several $\mathrm{K}^{+}$ channel subtypes across embryonic development. Similar findings have been reported for the whole brain (Hallows and Tem- 
pel, 1998) and in rat cochlear nucleus during postnatal development (Caminos et al., 2005; Bortone et al., 2006). Alternatively, in chick tangential vestibular nucleus Kv1 channels decrease in expression between E16 and posthatch time points (Popratiloff et al., 2003). Thus, $\mathrm{LVA} \mathrm{K}^{+}$channels can be regulated in a variety of ways during development, having major impacts on the physiological responses of the neuron.

Voltage-activated currents are integral to shaping EPSPs and APs, controlling the temporal fidelity of neurons responsible for time coding (Reyes et al., 1994; Rathouz and Trussell, 1998; Parameshwaran et al., 2001). In general, blockade or absence of Kv1-type channels increases membrane time constant, resulting in broader APs (Rathouz and Trussell, 1998; Brew et al., 2003). In MNTB, the all-important temporal precision of the auditory system is disrupted in mice lacking the Kv1.1 protein (Gittelman and Tempel, 2006). Mature NM neurons exposed to DTX-I fire multiple spikes in response to depolarizing current injection (Rathouz and Trussell, 1998). This is similar to the behavior of immature NM neurons reported here. In nucleus angularis, the other subdivision of the avian cochlear nucleus, DTX-I application to posthatch neurons results in spiking similar to embryonic neurons (Fukui and Ohmori, 2003). In chick tangential vestibular nuclei, developmental decreases in Kv1-type channels correlate with a switch from phasic to tonic firing in response to depolarization (Shao et al., 2006). Thus, developmental $\mathrm{K}^{+}$current regulation is key to modulating EPSPs and APs in neurons of the auditory and other systems.

LVA $\mathrm{K}^{+}$currents in NM neurons also shape depolarizing GPSPs in NM. DTX-I application slows, and eliminates the biphasic nature of GPSP decay in mature NM neurons (Monsivais and Rubel, 2001). GPSP trains tonically activate strong outward currents in NM (Monsivais et al., 2000). Monsivais and Rubel (2001) also describe excitation by miniature GPSPs during DTX-I application, with spikes evoked by each GABAergic event. Our observations substantiate this finding, demonstrating that LVA $\mathrm{K}^{+}$channel blockade results in hyperpolarization of AP voltage threshold and disrupts spike threshold accommodation during slow GPSP depolarization. Finally, DTX-I blocks shunting of excitatory inputs, reverting GABAergic depolarizations from inhibitory to summating. Thus, developmental increases in Kv1-type currents are essential for normal physiological responses to both excitation and inhibition in mature NM neurons and highlight the importance of coupling between synaptic and voltage-activated conductances as these neurons integrate inputs.

It is notable that responses of mature NM neurons during DTX-I application did not perfectly mimic those of immature neurons. Other voltage-dependent properties of the membrane not defined by this study are changing between E14 and E18. For example, Kv1 current increases would oppose the AP voltage threshold hyperpolarization between these ages. Current pulse summation with either GPSP trains or voltage steps was not as great in DTX-I exposed E18 neurons as in control E14 neurons. This is in part because of dendrotoxin tissue penetration problems (Gittelman and Tempel, 2006). Complete block of Kv1 currents may not be accomplished for tens of minutes, and residual $\mathrm{Kv} 1$ currents are likely shunting some excitation. Other sources of shunting such as DTX-I-insensitive $\mathrm{K}^{+}$currents may increase between E14 and E18 as well. However, our data make clear that E14-E18 Kv1 current growth is necessary to switch GABAergic inputs from enhancing excitability to strongly inhibiting mature NM neurons.

In immature $\mathrm{NM}$ neurons, $E_{\mathrm{GABA}}$ is negative to $\mathrm{AP}$ voltage threshold, so GPSPs do not evoke spikes unless summing with other depolarizing inputs. Thus, GABA is not an excitatory neurotransmitter at E14 despite enhancing the excitability of the neuron. In E18 NM neurons, $E_{\mathrm{GABA}}$ and AP voltage threshold are nearly identical. We observed GPSP-evoked APs in a small number (2 of 13) of E18 neurons under control conditions. This phenomenon has been reported previously, with frequency of occurrence varying between studies ( $\mathrm{Lu}$ and Trussell, 2001; Lu et al., 2005). Lu and Trussell (2001) reported $E_{\mathrm{GABA}}$ values that were depolarized compared with those reported here (see Results). Their reported $\mathrm{E}_{\mathrm{GABA}}$ values were well depolarized to their reported values of AP threshold $(-39 \pm 2 \mathrm{mV})$. This likely accounts for the greater frequency of GABA-evoked spikes that $\mathrm{Lu}$ and Trussell (2001) reported. Monsivais and Rubel (2001) and the present report illustrate the essential nature of Kv1 currents in making GABA an inhibitory neurotransmitter in mature NM neurons despite the similarity between $E_{\mathrm{GABA}}$ and AP voltage threshold. Additional mechanisms exist to control the occurrence of GABA-evoked spikes in NM. For example, activation of $\mathrm{GABA}_{\mathrm{B}}$ receptors, present in avian cochlear nuclei, has been shown to modulate $\mathrm{GABA}_{\mathrm{A}} \mathrm{R}$ events, minimizing GPSP-evoked APs (Burger et al., 2005; Lu et al., 2005).

In conclusion, changes in LVA Kv1 currents reverse the input/ output transform of the responses of NM neurons to GABAergic input across an important period of development immediately after the onset of hearing in the chick embryo. Intracellular $\mathrm{Cl}^{-}$ concentration, implicated in the developmental switch from GABAergic excitation to inhibition in other CNS systems, remains stable in these neurons into maturity. Synaptic conductances also remain relatively constant across this developmental period and, thus, are not responsible for the reversal. This study identifies changes to intrinsic voltage-dependent properties of neurons as an alternative to $E_{\mathrm{Cl}^{-}}$shift as a mechanism for developmental conversion in neural responses to GABAergic input from excitation to inhibition.

\section{References}

Akaiki N (1996) Gramicidin perforated patch recording and intracellular chloride activity in excitable cells. Prog Biophys Mol Biol 65:251-264.

Balakrishnan V, Becker M, Lohrke S, Nothwang HG, Guresir E, Friauf E (2003) Expression and function of chloride transporters during development of inhibitory neurotransmission in the auditory brainstem. J Neurosci 23:4134-4145.

Banke TG, McBain CJ (2006) GABAergic input onto CA3 hippocampal interneurons remains shunting through development. J Neurosci 26:11720-11725.

Ben-Ari Y, Cherubini E, Corradetti R, Gaiarsa JL (1989) Giant synaptic potentials in immature rat CA3 hippocampal neurones. J Physiol (Lond) 416:303-325.

Bormann J, Hamill OP, Sakmann B (1987) Mechanisms of anion permeation through channels gated by glycine and gamma-aminobutyric acid in mouse cultured spinal neurones. J Physiol (Lond) 385:243-286.

Bortone DS, Mitchell K, Manis PB (2006) Developmental time course of potassium channel expression in the rat cochlear nucleus. Hear Res 211:114-125.

Brew HM, Hallows JL, Tempel BL (2003) Hyperexcitability and reduced low threshold potassium currents in auditory neurons of mice lacking the channel subunit Kv1.1. J Physiol (Lond) 548:1-20.

Burger RM, Pfeiffer JD, Westrum LE, Bernard A, Rubel EW (2005) Expression of $\mathrm{GABA}_{\mathrm{B}}$ receptor in the avian auditory brainstem: ontogeny, afferent deprivation, and ultrastructure. J Comp Neurol 489:11-22.

Caminos E, Vale C, Lujan R, Martinez-Galan JR, Juiz JM (2005) Developmental regulation and adult maintenance of potassium channel proteins (Kv1.1 and Kv1.2) in the cochlear nucleus of the rat. Brain Res 1056:118-131.

Chang EH, Kotak VC, Sanes DH (2003) Long-term depression of synaptic inhibition is expressed postsynaptically in the developing auditory system. J Neurophysiol 90:1479-1488. 
Chebib M, Johnston GA (1999) The "ABC" of GABA receptors: a brief review. Clin Exp Pharmacol Physiol 26:937-940.

Cherubini E, Rovira C, Gaiarsa JL, Corradetti R, Ben-Ari Y (1990) GABA mediated excitation in immature rat CA3 hippocampal neurons. Int J Dev Neurosci 8:481-490.

Duzhyy DE, Sakai Y, Sokolowski BH (2004) Cloning and developmental expression of Shaker potassium channels in the cochlea of the chicken. Brain Res Mol Brain Res 121:70-85.

Eccles JC, Eccles RM, Magni F (1961) Central inhibitory action attributable to presynaptic depolarization produced by muscle afferent volleys. J Physiol (Paris) 159:147-166.

Edwards DH (1990a) Mechanisms of depolarizing inhibition at the crayfish giant motor synapse. I. Electrophysiology Neurophysiol 64:532-540.

Edwards DH (1990b) Mechanisms of depolarizing inhibition at the crayfish giant motor synapse. II. Quantitative reconstruction. J Neurophysiol 64:541-550.

Ehrlich I, Lohrke S, Friauf E (1999) Shift from depolarizing to hyperpolarizing glycine action in rat auditory neurones is due to age-dependent $\mathrm{Cl}^{-}$ regulation. J Physiol (Lond) 520:121-137.

Fukui I, Ohmori H (2003) Developmental changes in membrane excitability and morphology of neurons in the nucleus angularis of the chicken. J Physiol (Lond) 548:219-232.

Fukui I, Ohmori H (2004) Tonotopic gradients of membrane and synaptic properties for neurons of the chicken nucleus magnocellularis. J Neurosci 24:7514-7523.

Ge S, Goh EL, Sailor KA, Kitabatake Y, Ming GL, Song H (2006) GABA regulates synaptic integration of newly generated neurons in the adult brain. Nature 439:589-593.

Gittelman JX, Tempel BL (2006) Kv1.1 containing channels are critical for temporal precision during spike initiation. J Neurophysiol 96:1203-1214.

Graham B, Redman S (1994) A simulation of action potentials in synaptic boutons during presynaptic inhibition. J Neurophysiol 71:538-549.

Hallows JL, Tempel BL (1998) Expression of Kv1.1, a Shaker-like potassium channel, is temporally regulated in embryonic neurons and glia. J Neurosci 18:5682-5691.

Hyson RL, Reyes AD, Rubel EW (1995) A depolarizing inhibitory response to GABA in brainstem auditory neurons of the chick. Brain Res 677:117-126.

Jackson H, Hackett JT, Rubel EW (1982) Organization and development of brain stem auditory nuclei in the chick: ontogeny of postsynaptic responses. J Comp Neurol 210:80-86.

Jhaveri S, Morest DK (1982) Sequential alterations of neuronal architecture in nucleus magnocellularis of the developing chicken: a Golgi study. Neuroscience 7:837-853.

Kakazu Y, Akaike N, Komiyama S, Nabekura J (1999) Regulation of intracellular chloride by cotransporters in developing lateral superior olive neurons. J Neurosci 19:2843-2851.

Kennedy D, Calabrese RL, Wine JJ (1974) Presynaptic inhibition: primary afferent depolarization in crayfish neurons. Science 186:451-454.

Kriegstein AR, Owens DF (2001) GABA may act as a self-limiting trophic factor at developing synapses. Sci STKE 95:PE1.

Koyano K, Funabiki K, Ohmori H (1996) Voltage-gated ionic currents and their roles in timing coding in auditory neurons of the nucleus magnocellularis of the chick. Neurosci Res 26:29-45.

Lu T, Trussell LO (2000) Inhibitory transmission mediated by asynchronous transmitter release. Neuron 26:683-694.
Lu T, Trussell LO (2001) Mixed excitatory and inhibitory GABA-mediated transmission in chick cochlear nucleus. J Physiol (Lond) 535:125-131.

Lu Y, Monsivais P, Tempel BL, Rubel EW (2004) Activity-dependent regulation of the potassium channel subunits Kv1.1 and Kv3.1. J Comp Neurol 470:93-106.

Lu Y, Burger RM, Rubel EW (2005) GABA(B) receptor activation modulates $\mathrm{GABA}(\mathrm{A})$ receptor-mediated inhibition in chicken nucleus magnocellularis neurons. J Neurophysiol 93:1429-1438.

Magnusson AK, Kapfer C, Grothe B, Koch U (2005) Maturation of glycinergic inhibition in the gerbil medial superior olive after hearing onset. J Physiol (Lond) 568:497-512.

McBain CJ, Fisahn A (2001) Interneurons unbound. Nat Rev Neurosci 2:11-23.

Monsivais P, Rubel EW (2001) Accommodation enhances depolarizing inhibition in central neurons. J Neurosci 21:7823-7830.

Monsivais P, Yang L, Rubel EW (2000) GABAergic inhibition in nucleus magnocellularis: implications for phase locking in the avian auditory brainstem. J Neurosci 20:2954-2963.

Oertel D (1983) Synaptic responses and electrical properties of cells in brain slices of the mouse anteroventral cochlear nucleus. J Neurosci 3:2043-2053.

Okada M, Onodera K, Van Renterghem C, Sieghart W, Takahashi T (2000) Functional correlation of $\mathrm{GABA}_{\mathrm{A}}$ receptor $\alpha$ subunits expression with the properties of IPSCs in the developing thalamus. J Neurosci 20:2202-2208.

Owens DF, Boyce LH, Davis MB, Kriegstein AR (1996) Excitatory GABA responses in embryonic and neonatal cortical slices demonstrated by gramicidin perforated-patch recordings and calcium imaging. J Neurosci 16:6414-6423.

Parameshwaran S, Carr CE, Perney TM (2001) Expression of the Kv3.1 potassium channel in the avian auditory brainstem. J Neurosci 21:485-494.

Popratiloff A, Giaume C, Peusner KD (2003) Developmental change in expression and subcellar localization of two shaker-related potassium channel proteins (KV1.1 and KV1.2) in the chick tangential vestibular nucleus. J Comp Neurol 461:466-482.

Rathouz M, Trussell L (1998) Characterization of outward currents in neurons of the avian nucleus magnocellularis. J Neurophysiol 80:2824-2835.

Reyes AD, Rubel EW, Spain WJ (1994) Membrane properties underlying the firing of neurons in the avian cochlear nucleus. J Neurosci 14:5352-5364.

Rivera C, Voipio J, Payne JA, Ruusuvuori E, Lahtinen H, Lamsa K, Pirvola U, Saarma M, Kaila K (1999) The $\mathrm{K}^{+} / \mathrm{Cl}^{-}$co-transporter KCC2 renders GABA hyperpolarizing during neuronal maturation. Nature 397:251-255.

Rubel EW, Smith DJ, Miller LC (1976) Organization and development of brain stem auditory nuclei of the chicken: ontogeny of n. magnocellularis and n. laminaris. J Comp Neurol 166:469-489.

Shao M, Hirsch JC, Peusner KD (2006) Emergence of action potential generation and synaptic transmission in vestibular nucleus neurons. J Neurophysiol 96:1215-1226.

Slee SJ, Higgs MH, Fairhall AL, Spain WJ (2005) Two-dimensional time coding in the auditory brainstem. J Neurosci 25:9978-9988.

Staley KJ, Soldo BL, Proctor WR (1995) Ionic mechanisms of neuronal excitation by inhibitory $\mathrm{GABA}_{\mathrm{A}}$ receptors. Science 269:928-929.

Trussell LO (1997) Cellular mechanisms for preservation of timing in central auditory pathways. Curr Opin Neurobiol 7:487-492. 